\title{
VARIABLE NEIGHBORHOOD SEARCH BASED ALGORITHMS FOR CROSSDOCK TRUCK ASSIGNMENT
}

\author{
Cécilia Daquin*, Hamid Allaoui, Gilles Goncalves and Tienté Hsu
}

\begin{abstract}
To operate a cross-dock successfully, an efficient assignment of trucks to docks is one of the key decisions. In this paper, we are interested in the cross-dock assignment of trucks to docks problem, where the number of trucks exceeds the number of docks. The objective is to minimize the cost of transferring goods within the cross-dock while avoiding delivery penalties. This problem being NP-hard, we use Variable Neighborhood Search metaheurisitc (VNS) to solve it approximately. More specifically, we conduct a structured empirical study to compare several VNS configurations and to find which is/are the most effective for this cross-dock problem. In this work, first we analyze the way the search strategy and the neighborhood operators can be combined in a VNS framework according to their efficiency within a local search. Then the best configurations are tested within three VNS variants, namely Basic VNS (BVNS), General VNS (GVNS) using Basic VND (B-VND) and GVNS using Union VND (U-VND) according to the number of used operators and the order of applying these operators. Finally we evaluate the influence of the stopping criterion within these variants. Some significant differences among these configurations are shown and illustrated by conducting the Friedman test.
\end{abstract}

Mathematics Subject Classification. 90B06.

Received September 23, 2019. Accepted August 3, 2020.

\section{INTRODUCTION}

Cross-docking is a common logistics practice in industry where goods are received from suppliers in inbound trucks and loaded directly without storage into outbound trucks to customers. It enables to reduce operational costs while shortening delivery time and increasing goods flow (Fig. 1). A cross-dock includes various docks to which trucks can be assigned for unloading and loading operations. Inbound trucks are assigned to docks to unload received goods from suppliers. Then goods are immediately transferred to appropriate docks where outbound trucks are assigned. Finally, goods are loaded for delivering customers. Once an inbound truck is completely unloaded or an outbound truck is completely loaded, the dock is available for another truck. The problem is how to assign docks to trucks while synchronizing the arrival and the departure of cargos This property allows cost and delivery lead-time to be reduced. Moreover, this practice improves resource utilization by getting full trucks. All these advantages make cross-docking an interesting logistics practice for many companies. However, flows have to be well managed to keep these benefits achievable.

Keywords. Cross-dock, metaheuristics, local search, variable neighborhood search, variable neighborhood descent.

Univ. Artois, UR 3926, Laboratoire de Génie Informatique et d'Automatique de l'Artois (LGI2A), F-62400 Béthune, France.

*Corresponding author: cecilia.daquin@univ-artois.fr 


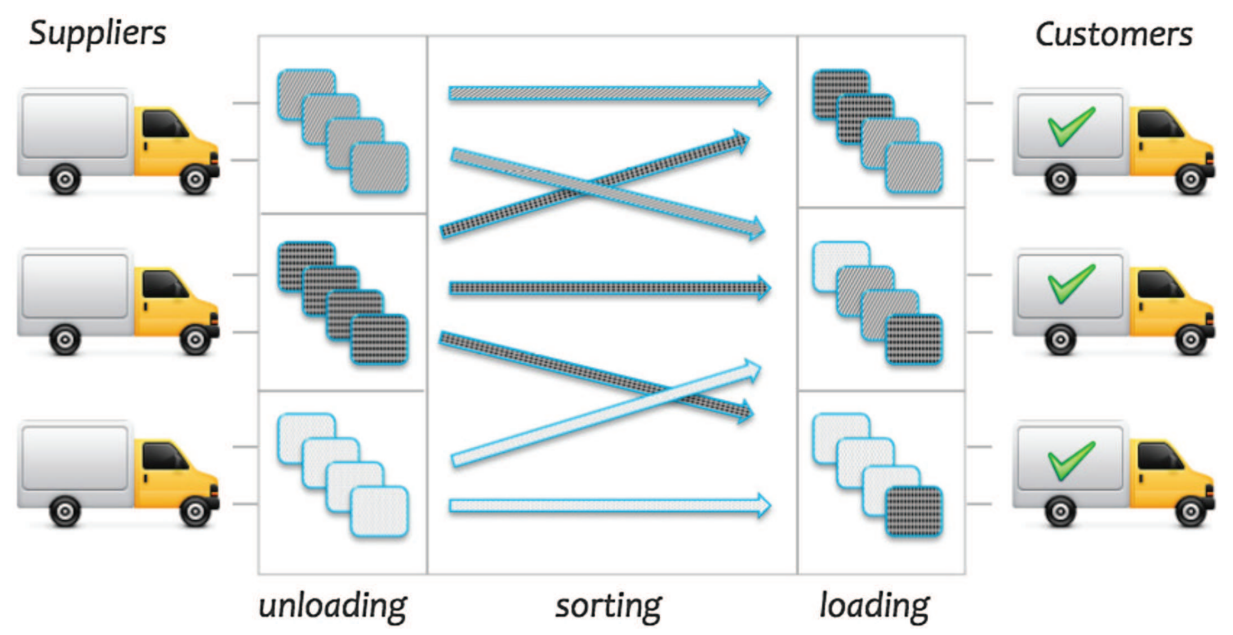

FIGURE 1. Schematic representation of a cross-dock.

Many decisions have to be taken by the practitioners about the use and implementation of cross-docking. These decisions have to be decided cautiously, because they have a major impact on the cross-docking efficiency. The first issue is to fix the location of the cross-dock and its layout. The network has to be designed so as to determine the goods flows through the network. Afterwards, the practitioners have to decide on routing of trucks before and after passing through the cross-dock. Once arriving at the cross-dock, the assignment of trucks to docks has to be addressed. Finally, resources inside the cross-dock have to be scheduled, and the storage area has to be located. In this paper, we focus on the assignment of trucks to docks in order to optimize the sum of operational cost and penalty cost. Because we assume that the number of trucks exceeds the number of docks, some penalties have to be paid when trucks cannot be scheduled in the current horizon. For this problem, we use the Integer Linear Programming (ILP) model proposed by Miao et al. [21] and propose an amendment to this model. According to our knowledge, this amended model was not considered in the literature yet. Since this combinatorial optimization problem is known to be NP-hard [21], we use several VNS variants to find optimal or near optimal solutions to our model.

The VNS algorithm consists of three steps which are executed until the stopping criterion is reached: the shaking step, the improvement procedure and the neighborhood change step (cf. Sect. 5). Following this basic scheme, different VNS variants can be obtained by choosing different settings: number and/or order of neighborhoods, the search strategy, the descent phase configuration, the stopping condition, etc. Thus, in this paper, we are interested in these different possible configurations of VNS in order to find the most effective for the studied cross-docking problem (Fig. 2). First, we focus on several neighborhood operators and the way they can be combined as VNS inputs to solve this problem. We propose four neighborhood operators. One of them is used by Lim et al. [20] and we develop the other ones for the cross-dock problem. We test and compare them one by one within a local search using two different search strategies. The aim is to highlight their characteristics and their efficiency in order to operate VNS algorithms much better as possible. Then, according to the results, we test several strategies for combining the operators in VNS structures. These strategies differ by the number and the order of the neighborhood operators. Regarding the VNS structure, we are interested in widely used variants of VNS method: the basic VNS (BVNS) that uses a local search in a descent phase, the general VNS (GVNS) that uses a basic VND (Variable Neighborhood Descent) and the GVNS that uses an union VND. After detecting the best strategy for the BVNS, we use it within the two GVNS variants and we compare the performances. Finally, we are interested in the impact of stopping criterion within VNS algorithm over the results. In each step of our empirical study, the performance of the tested algorithms is evaluated on a set of 


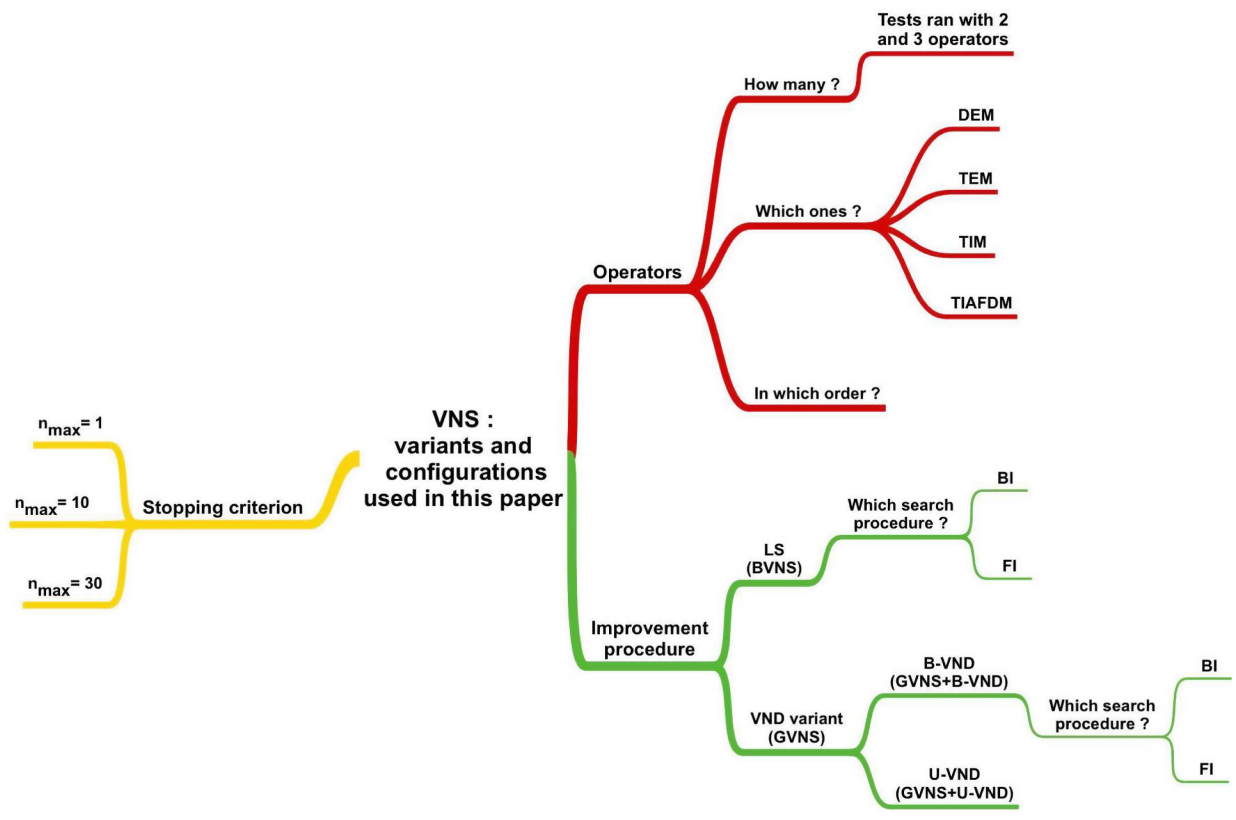

Figure 2. Overview of the VNS configurations tested in this paper.

instances of the cross-docking problem and compared with the performance of CPLEX solver. However, we couldn't compare our results with ones of Miao et al. [21] since their benchmarks are not available, and both models are slightly different (objective functions differ). Finally, the Friedman test is conducted to analyse the statistical significance of the proposed algorithms performance.

In the remainder of this paper, a literature review is presented in Section 2. Afterwards a formal description and the ILP model of the problem are found in Section 3. Sections 4 and 5 present the local search, the VNS variants used to solve this problem, their possible configurations and the proposed neighborhood operators. Computational results are given and analysed in Section 6. Finally, our results are summarized and possible future research is suggested in Section 7.

\section{LiterATURE REVIEW}

The cross-docking problem is well studied in recent literature. Boysen and Fliedner [6] provided a classification and a literature review for the truck scheduling problem. Van Belle et al. [35] presented a thorough review of papers about several problems related to cross-docking. Lately, Ladier and Alpan [17] compared the articles related to cross-docking operations found in the literature with the industrial practices captured through visits to cross-docks and interviews with their managers. Some papers discuss factors that influence the suitability of cross-docking. Apte and Viswanathan [3] examined features and techniques of effective cross-docking compared with traditional distribution. Van Belle et al. [35] provided guidelines for a successful implementation of crossdocking. Buijs et al. [7] proposed a framework specifying the interdependencies between different aspects of cross-docking problem and presented a new general classification scheme for cross-docking research based on the inputs and outputs for each problem aspect. Thus, during the design and operational phase of cross-docks, many decisions have to be taken.

In cross-docking, the temporary storage is an important element to manage. Indeed, products unloaded from trucks can be stored few hours at most while waiting for a truck to arrive. Thus, a staging strategy have to be decided. Taylor and Noble [32] examined three material staging alternatives in various cross-dock environments. 
They suggested that staging needs can vary depending on different staging methods and the scenarios considering a range of demand types. Sandal [31] extended this study and examined several staging strategies in order to determine which one is the most appropriate in a cross-docking operation according to freight attributes and container loading requirements. Vis and Roodbergen [36] sought to determine temporary storage locations for incoming freight in order to minimize the travel distances of goods. Their method consisted of a network formulation that incorporated loading and unloading dock door locations, travel distances and available storage space in the facility. Experimental results showed the proposed algorithm can reduce the total travel distance up to about $40 \%$.

One of the key decisions is the dock assignment for inbound and outbound trucks. An efficient dock assignment can increase the performance of the cross-dock and can reduce the shipment delays, the operational time and costs in the cross-dock, among other things. So, dock door assignment problem tries to provide the best assignment for trucks to docks at a given point in time. This problem considers the number of trucks being less than or equal to the number of docks, and the time dimension are not taken into consideration. The purpose is to assign each truck to a different door, considering the spatial dimension, i.e. the location of the doors. Tsui and Chang [33] formulated the dock door assignment problem as a bilinear programming problem where the objective function is nonlinear that tries to minimize the total distance traveled by the forklifts. A branch-andbound algorithm is proposed to solve this problem. Cohen and Keren [9] suggested another optimal formation that is a non-linear MIP model and a heuristic approach for assigning cross-dock doors to trailers. Nassief et al. [28] formulated the cross-dock door assignment problem as a mixed integer programming which embedded into a Lagrangian relaxation that exploited the structure of the problem to obtain lower and upper bounds on the optimal solution. This approach allowed to obtain solutions of instances more difficult. Nassief et al. [29] proposed two new MIP models for this problem and compared the results of their computational and analytical experiments with existing ones.

Dock assignment problems do not take into consideration temporal constraints, so it is not possible to assign multiple trucks to one dock sequentially. Yet, other problems consider more trucks than docks and seek to determine the succession of the arriving and departing trucks at the docks. In this case, only the temporal dimension is taken into consideration, without spatial constraints. Then, the so-called truck scheduling problem considers dock doors as resources that have to be scheduled over time. Note that the dock assignment problem is part of the truck scheduling problem: over a first phase, the order and/or the time of trucks assignment is decided in order to choose a dock for each truck next. Chen and Lee [8] studied a two-machine cross-docking flow shop scheduling problem in which an operation on the second machine cannot be processed unless the operation on the first machine has been completed. Their purpose was to sequence both the inbound and outbound carriers to minimize the makespan. In their paper, they proved that the problem is strongly NP-hard and developed a branch-and-bound algorithm to solve it optimally. Yu and Egbelu [37] studied a cross-docking system where a temporary storage area is located in front of the shipping dock. They sought to find the best truck docking or best scheduling sequence for both inbound and outbound trucks to minimise the makespan. In their paper, a mixed integer programming model is formulated, and a heuristic algorithm is proposed to solve large problem instances. For the same problem, Vahdani and Zandieh [34] applied five metaheuristics to solve it including a genetic algorithm, tabu search, simulated annealing and variable neighborhood search. Their experimental study showed these metaheuristics resulted clearly better solution than the ones obtained by the heuristic in [37]. Boloori Arabani et al. [5] studied the same problem and developed also five metaheuristics: a genetic algorithm, tabu search, particle swarm optimization, ant colony optimization and differential evolution. Alpan et al. [2] proposed a bounded dynamic approach to schedule inbound and outbound trucks in a multiple receiving and shipping cross-dock environment. Alpan et al. [1] developed several heuristics to find the best schedule to minimize the sum of inventory holding and truck replacement costs. Lim et al. [20] considered a problem with time windows and capacity constraints, i.e. trucks are assigned to docks within its time window to unload and load the carried merchandises through the cross-dock with a limited capacity of storage. The objective was to minimize the total shipping distances between docks. In their paper, the problem is formulated as an integer programming model, then proposed a tabu search and a genetic algorithm to solve it. Later, they 
extended this problem by focusing also on the operational time between docks [19]. Their new purpose was to minimize the operational cost, which is calculated according to the distance and the time travel between docks, and the penalty cost for unfulfilled shipments. This problem is formulated as an integer programming model and the authors proposed a genetic algorithm and finally a tabu search in [21] to solve it optimally. Miao et al. [22] proposed a new model in which the capacity constraint didn't take into consideration anymore, and distinguished the inbound trucks and docks from the outbound trucks and docks. They developed an adaptive tabu search to solve it. Gelareh et al. [15] proposed a different model for the same problem as in [21] and provided an efficient branch-and-cut algorithm to solve it in a reasonable computational time. Recently, Miao et al. [23] proposed a new model in which several practical constraints are simultaneously considered such as the capacity of trucks and of storage area, the time windows of trucks and the operational time within the crossdock. They proposed a two stage genetic algorithm to solve it. These last problems are similar to problems of gate assignment in airports. In the literature, some papers dealt with this subject $[4,11,12,18,30]$. Because the gate assignment problem is NP-hard [30] and it is a special case of the problem studied in [21], the latter showed their problem is NP-hard. Dondo and Cerdá [13] developed a mixed integer linear programming formulation for the vehicle routing problem with cross-docking to find the routing and scheduling of a mixed fleet, the truck docking sequence, the dock door assignment and the travel time to move the goods through the crossdock. Mohtashami et al. [27] proposed a multi-objective mathematical model that minimizes the makespan, transportation costs and the number of truck trips in the entire supply chain. To solve it, they developed two evolutionary metaheuristic algorithms: the non-dominated sorting genetic algorithm and the multi objective particle swarm optimization. Fonseca et al. [14] proposed a lagrangian relaxation methodolody to solve a truck scheduling problem in a crossdock with parallel-docks. The objective is to minimize the makespan. Mohtashami [26] studied a cross-docking problem where there is temporary storage in front of the shipping dock and the shipping trucks can move in and out of the dock repeatedly. In fact, after the shipping truck loads some of its needed products, it can choose between two scenarios: either more products are loaded into the current shipping truck, or the current shipping truck is moved out from the shipping dock and another shipping truck is moved to the shipping dock to load its products. In this case, an outbound truck can load some of its needed products from shipping dock, leaves the dock for another outbound truck, waits and goes into the shipping dock again to load all or part of its remaining product items. Its purpose was to find the optimal/near optimal solution for sequencing inbound and outbound trucks to minimize the total operational cost. To solve this problem, the author developed a genetic algorithm-based method.

For the best of our knowledge, the proposed amendment to the model presented in Miao et al. [21] is considered for the first time. Since the problem is still NP-hard, it is unlikely to find optimal solutions for big instances in a reasonable computational time. It is why we propose in this paper several VNS metaheuristic variants to get approximate solutions. Hence, the main purpose here is to deliver a wide structured experimental study and a sensitivity analysis to compare several VNS configurations. The aim is to investigate some significant differences among them and to highlight the most effective for the studied problem.

\section{Problem statement}

Our study focuses on assignment of trucks to docks within a cross-dock under time window constraints as defined in $[20,21]$. Indeed, we seek to assign trucks to docks in order to minimize the sum of operational cost and penalty cost. The operational cost is the cost of transferring pallets from inbound docks to outbound docks. The penalty cost is the cost of not being able to transfer pallets from dock to dock. This is due to not assigning corresponding inbound truck or outbound truck in the required time window. In this case a negotiation could be engaged with the truck provider in the possibility to reassign it outside the required time window. We consider the problem whenever the number of trucks exceeds the number of available docks. The problem data are:

- The time window $\left[a_{i} ; d_{i}\right]$ of each truck $i$, i.e., $a_{i}$ denotes the time instant in which truck $i$ arrives and is assigned to a dock and $d_{i}$ denotes the time instant in which truck $i$ leaves its assigned dock. So then, the arrival and departure times of each truck have already been set; 
- The cross-dock characteristics (number of docks, costs and time of transferring goods between docks and the maximum number of shipments that the warehouse can hold);

- The flow of shipments (the quantities and the penalty costs if goods are unfulfilled, the inbound and outbound trucks that have to transport goods).

Our purpose is to minimize the total cost, which is the sum of the cost of transferring goods between docks and the penalty cost. Therefore, we have to find the best dock assignments for trucks. Moreover, several constraints must be satisfied:

- A truck should be assigned to one dock during its time window, i.e. starting from its arriving time until its departure time. So then, it is necessary to ensure at least one dock is available during the time window of truck. If no dock is free, the latter will not be assigned and a penalty cost will be incurred for not transferred pallets;

- At any given time, only one truck can be assigned to a certain dock. If two trucks are assigned to the same dock, their respective time windows must not overlap;

- At any given time, the total number of goods inside the cross-dock cannot be greater than the maximum capacity of the warehouse (capacity constraint);

- The goods transfer process between two trucks has to take place between the arrival time of the inbound truck and the departure time of the outbound truck, while considering the time of transferring goods between two docks (precedence constraint).

Whenever a truck isn't assigned during its time window, a penalty cost is incurred for all the unfulfilled shipment by this truck.

Miao et al. [21] studied the same problem and proposed an IP model. We present in the following the same model except the objective for which we propose an amendment. Indeed, in the Miao et al.'s model, a transfer cost is incurred as soon as two docks are assigned, even if there is no shipment between these ones. We propose to add a new preprocessing variable $\sigma$ in the operational cost to take into account an incurred cost if and only if there are transferred pallets between assigned trucks.

The following notations that we use are the same as in [21]:

$N$ set of trucks arriving at and/or departing from the cross-dock.

$M$ set of docks available in the cross-dock.

$n$ total number of trucks.

$m$ total number of docks.

$a_{i}$ opening time of time window of truck $i(1 \leq i \leq n)$.

$d_{i}$ closing time of time window of truck $i(1 \leq i \leq n)$.

$t_{k, l}$ operational time for pallets from dock $k$ to dock $l(1 \leq k, l \leq m)$.

$f_{i, j}$ number of pallets moving from truck $i$ to truck $j(1 \leq i, j \leq n)$.

$c_{k, l}$ operational cost per unit time from dock $k$ to dock $l(1 \leq k, l \leq m)$.

$p_{i, j}$ penalty cost per unit shipment from truck $i$ to truck $j(1 \leq i, j \leq n)$.

$C$ capacity of cross-dock, i.e. the maximum number of shipment the cross-dock can hold at each time.

This binary pre-processing parameter is also defined:

$x_{i, j}=1$ iff truck $i$ departs no later than truck $j$ arrives, i.e. iff $d_{i} \leq a_{j}$; 0 otherwise

We propose to add this new pre-processing parameter:

$\sigma_{i, j}=1$ iff $f_{i, j}>0$, i.e. if shipment has to be transferred from truck $i$ to truck $j ; 0$ otherwise.

The decision variables are as follows:

$$
\begin{aligned}
y_{i, k} & =\left\{\begin{array}{l}
1 \text { if truck } i \text { is assigned to dock } k \\
0 \text { otherwise }
\end{array}\right. \\
z_{i, j, k, l} & =\left\{\begin{array}{l}
1 \text { if truck } i \text { is assigned to dock } k \text { and truck } j \text { is assigned to dock } l \\
0 \text { otherwise }
\end{array}\right.
\end{aligned}
$$


Through a pre-processing step, we set the parameters $\sigma_{i, j}$ and $x_{i, j}(1 \leq i, j \leq n)$. Moreover, in order to make data consistent, we give some assumptions as in [21]:

- $f_{i, j} \geq 0$, iff $d_{j} \geq a_{i}(1 \leq i, j \leq n)$, otherwise $f_{i, j}=0$. In other word, truck $i$ will transfer some shipment to truck $j$ iff truck $j$ departs no earlier than truck $i$ arrives. Note that even if $d_{j} \geq a_{i}, f_{i, j}$ can be equal to zero i.e. truck $i$ has no shipment to transfer to truck $j$;

- $a_{i}<d_{i}(1 \leq i, j \leq n)$ which means that for each truck, the arrival time must be strictly smaller than the departure time;

- $n>m$ because we consider the problem when the number of trucks exceeds the number of available docks;

- Capacity $C$ is defined as follows: when truck $i$ comes, then it will consume $\sum_{k=1}^{m} \sum_{l=1}^{m} \sum_{j=1}^{n} f_{i, j} z_{i, j, k, l}$ units of capacity whenever $i$ is assigned. Respectively, when truck $j$ leaves its dock, then $\sum_{k=1}^{m} \sum_{l=1}^{m} \sum_{i=1}^{n} f_{i, j} z_{i, j, k, l}$ units of capacity are released;

- Sort all the $a_{i}$ and $d_{i}$ in an increasing order $\left(1 \leq i, j \leq n\right.$ ), and let $t_{r}$ (with $r$ an integer such that $1 \leq r \leq 2 n$ ) correspond to these $2 n$ number such that $t_{1} \leq t_{2} \leq \ldots \leq t_{2 n}$. Using these notations, we can easily formulate the capacity constraint afterwards.

In order to minimize the total cost, which is the sum of the cost of transferring goods and the penalty cost, we propose the following ILP model:

$$
\min \sum_{k=1}^{m} \sum_{l=1}^{m} \sum_{i=1}^{n} \sum_{j=1}^{n} c_{k, l} t_{k, l} \sigma_{i, j} z_{i, j, k, l}+\sum_{i=1}^{n}\left(\sum_{j=1}^{n} p_{i, j} f_{i, j}\left(1-\sum_{k=1}^{m} \sum_{l=1}^{m} z_{i, j, k, l}\right)\right)
$$

s.t.

$$
\begin{aligned}
& \sum_{k=1}^{m} y_{i, k} \leq 1(1 \leq i \leq n) \\
& z_{i, j, k, l} \leq y_{i, k}(1 \leq i, j \leq n, 1 \leq k, l \leq m) \\
& z_{i, j, k, l} \leq y_{j, l}(1 \leq i, j \leq n, 1 \leq k, l \leq m) \\
& y_{i, k}+y_{j, l}-1 \leq z_{i, j, k, l}(1 \leq i, j \leq n, 1 \leq k, l \leq m) \\
& x_{i, j}+x_{j, i} \geq z_{i, j, k, k}(1 \leq i, j \leq n, i \neq j, 1 \leq k \leq m) \\
& \sum_{k=1}^{m} \sum_{l=1}^{m} \sum_{i \in\left\{i: a_{i} \leq t_{r}\right\}} \sum_{j=1}^{n} f_{i, j} z_{i, j, k, l} \\
& \quad-\sum_{k=1}^{m} \sum_{l=1}^{m} \sum_{i=1}^{n} \sum_{j \in\left\{j: d_{j} \leq t_{r}\right\}} f_{i, j} z_{i, j, k, l} \leq C(1 \leq r \leq 2 n) \\
& f_{i, j} z_{i, j, k, l}\left(d_{j}-a_{i}-t_{k, l}\right) \geq 0(1 \leq i, j \leq n, 1 \leq k, l \leq m) \\
& y_{i, k} \in\{0,1\}, y_{j, l} \in\{0,1\}, z_{i, j, k, l} \in\{0,1\}(1 \leq i, j \leq n, 1 \leq k, l \leq m) .
\end{aligned}
$$

In this formulation, the objective function (3.1) is composed of two terms: the total operational cost and the total penalty cost. In the first part, the total operational cost represents the transfer cost between two docks according to the transfer time for each shipment transfer. Therefore, compared to [21], we add the binary parameter $\sigma_{i, j}$ in the definition of the total operational cost. Then, whenever $\sigma_{i, j}=1$ i.e. when truck $i$ has to transfer shipments to truck $j$, the transfer cost is applied if trucks $i$ and $j$ are assigned. If $\sigma_{i, j}=0$, no transfer cost is added even if trucks $i$ and $j$ are assigned. In [21], this variable is not defined. In this case, with 
constraint (3.5), a transfer cost is incurred as soon as two trucks are assigned, even if there is no shipment between theses ones. The second part corresponds to penalty incurred when goods are not transferred from dock to dock because corresponding trucks are not assigned or precedence constraints are not checked.

Constraint (3.2) ensures that each truck can be assigned to one dock at most. Constraints (3.3) to (3.5) jointly define the variable $z$ which represent the logic relationship among $y_{i, k}, y_{j, l}$ and $z_{i, j, k, l}$. Constraint (3.6) guarantees that a dock cannot be occupied by more than one truck simultaneously. Constraint (3.7) defines the capacity constraint, i.e. at each event time (arrival and departure time of a truck), the total number of shipment inside the cross-dock cannot exceed the maximum capacity $C$. The last constraint (3.8) ensures that the shipment transfer between two trucks takes place within the time window of each truck while respecting precedence constraint between both of them.

\section{Proposed local Search Algorithm}

Before introducing the VNS framework, we first focus, in this section, on the neighborhood operators to analyse their efficiency within a local search. Local search (LS) is a simple procedure for heuristically solving combinatorial problems. This method is based on exploring the search space of candidate solutions until a "good" solution is found. The algorithm starts from an initial solution and then tries to "improve" it by moving to a neighborhood solution. A neighborhood structure $N(S, O p)$ is a set of solutions that differ only by one attribute or a combination of attributes from the current solution $S$. Neighborhood solutions are generated by applying a neighborhood operator $O p$ to the current solution and a strategy of neighborhood progress.

The local search algorithm takes as parameters an initial solution $S$, a neighborhood operator and a strategy of neighborhood progress. Through each iteration, the neighbors of the current solution are created in accordance with the chosen neighborhood operator $O p$. Once a solution $S^{\prime}$ is identified from this neighborhood structure $N(S, O p)$, it is compared against the current solution $S$. If the candidate solution $S^{\prime}$ is better, it becomes the current solution and the search continues. The search stops whenever the stopping criterion is reached, i.e. whenever the current solution is getting stuck in a local optimum (no more improvement). To select a solution from the neighborhood, two strategies are used generally. The Best Improvement (BI) consists in choosing the best neighbor (the one with the lowest objective function). The First Improvement (FI) chooses the first neighbor which improves the current solution. Note that it is not necessary to generate all the neighbors of the current solution whenever the strategy FI is used.

The algorithm of local search is given in Algorithm 1.

We generate the initial solution with a "First Come First Served" rule (FCFS): trucks are sorted by their arrival time and are assigned to the first free dock in this order. If no dock is free for a given truck, the latter

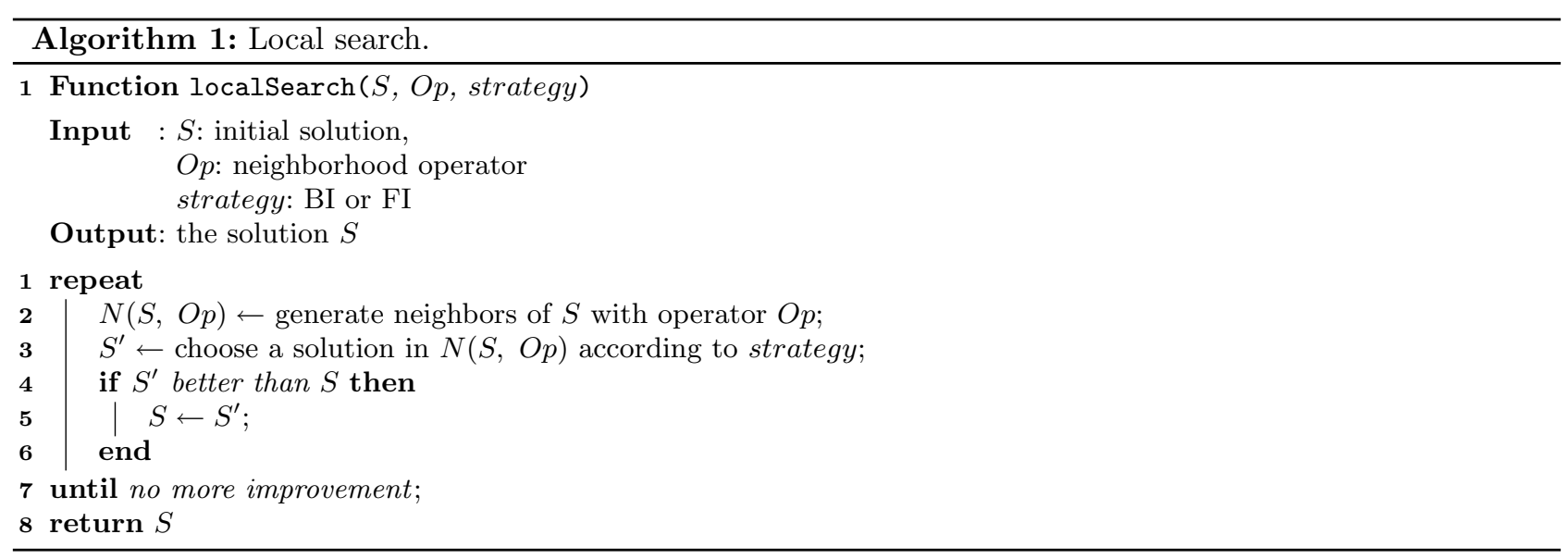




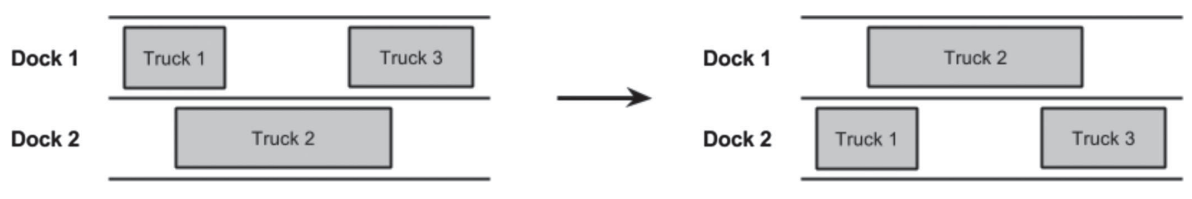

Figure 3. Dock Exchange Move operator (DEM).

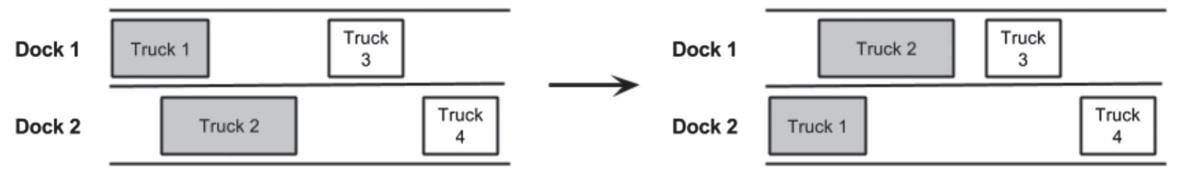

FiguRE 4. Truck Exchange Move operator(TEM).

will not be assigned. Hereafter, all the initial solutions used in the proposed algorithms are generated with this heuristic.

Neighborhood solutions are generated in accordance with a local search operator. In the rest of this study, the four operators presented below are used. Note that for each operator we examine all possible combinations.

\section{Dock Exchange Move (DEM)}

The first operator is a Dock Exchange Move (DEM): when considering two docks 1 and 2, all trucks assigned to dock 1 are moved to dock 2 and inversely. This operator enables to reduce the transferring costs between docks. Moreover, it only moves assigned trucks keeping their same time window and the same links of precedence as in the initial solution. Therefore, the produced solutions always satisfy all constraints of the problem. In the worst case, a solution can have $(m * m) / 2$ neighbors, where $m$ is the total number of docks (Fig 3).

\section{Truck Exchange Move (TEM)}

The second operator is a Truck Exchange Move (TEM): two trucks in a temporal conflict are exchanged. For example, let truck $i$ be assigned to dock 1 and truck $j$ assigned to dock 2 . If both trucks are in a temporal conflict, truck $i$ moves to dock 2 and truck $j$ to dock 1 . Two trucks are in a temporal conflict whenever their time windows have a shared part. The expected effects of this operator is to obtain larger free time windows (defragmentation) in order to receive new trucks. In addition, it enables to minimize transferring costs between docks. TEM can generate solutions that violate the time windows constraint. In this case, these invalid solutions are not added to the neighborhood. Thus, a solution can have $(n * m)$ neighbors in the worst case, where $n$ is the total number of trucks and $m$ the total number of docks (Fig 4 ).

\section{Truck Insert Move (TIM)}

The third operator is a Truck Insert Move (TIM): a truck $i$ (assigned or not) is assigned to another dock. If there were trucks in this dock that are in conflict with it, then the last trucks are rejected. For example, truck $i$ has to be assigned to dock 1. But the latter is taken over by truck $j$ during the time windows of truck $i$. Accordingly, truck $j$ is rejected to give a place to truck $i$. This operator enables to maximize the time windows, to reduce the transferring costs between docks and to minimize the penalty costs of non-delivering goods. TIM can generate solutions that break the time windows constraint and the capacity constraint of the cross-dock. Then, these invalid solutions are not added in the neighborhood. The neighborhood size will be equal to $(n * m)$ (Fig 5). This operator is used by Lim et al. [20].

\section{Truck Insert At Free Dock Move (TIAFDM)}

The last operator is a Truck Insert At Free Dock Move (TIAFDM): an assigned truck $i$ is moved to a free dock during the time window of $i$. The expected effect is to fill the holes in dock allocations and to obtain larger 


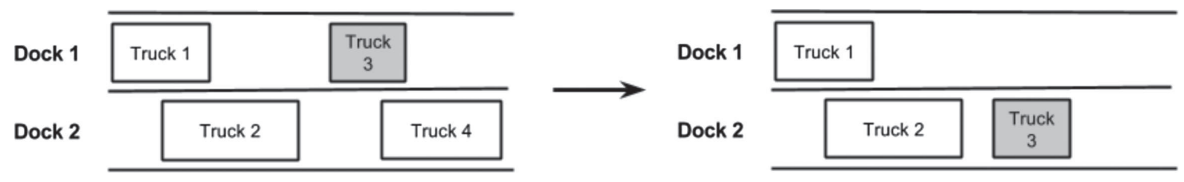

FiguRE 5. Truck Insert Move operator (TIM).

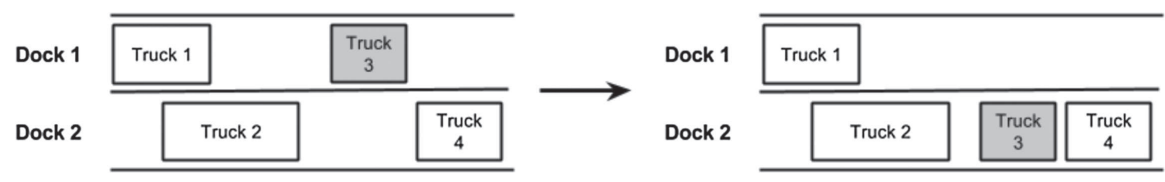

Figure 6. Truck Insert At Free Dock Move operator (TIAFDM).

time windows in order to receive a new truck. The solutions produced by this operator can violate the time windows constraint and the capacity constraint of the cross-dock. In this case, these invalid solutions aren't added in the neighborhood. In the worst case, this operator provides $(n * m)$ neighbors (Fig 6).

The main disadvantage of the local search is that the algorithm stops once the local optimum is reached and returns it. But, there is a big chance that a better solution exists in another neighborhood or in a larger neighborhood. Therefore, we only use this method to compare our proposed operators in order to combine it as better as possible in another metaheuristic: Variable Neighborhood Search (VNS).

\section{VARIABLE NEIGHBORHOOD SEARCH VARIANTS}

VNS is a metaheuristic proposed by Mladenovic̀ and Hansen [25]. Its main characteristic is to change systematically the neighborhood during the optimisation process for an optimal (or near-optimal) solution. This procedure is performed in both methods: get a minimum local, and get out of the valley. VNS is developed upon the following observations:

- A local optimum with respect to one neighborhood structure is not necessarily a local optimum for another neighborhood structure;

- A global optimum is a local optimum with respect to all the neighborhood structures;

- For many problems, a large majority of local optima with respect to one or several neighborhoods are close to each other relatively.

The first property incites to move in several neighborhood in order to find local optima with respect to all the neighborhoods used. The second property suggests using several neighborhoods whenever local optima found are of poor quality. The last property means that a local optimum often provides some information about the global one and so the search for the vicinity of the current solution has to be intensified. Overall, the VNS algorithm repeats three steps until the stopping criterion is reached: the shaking step, the improvement procedure and then the neighborhood change step. The first step generates the neighbors of the current solution $S$ within the current neighborhood operator and then a random solution $S^{\prime}$ is chosen among them. Thus, the shaking phase perturbs the local optimum found through the current iteration in order to provide a good starting point for the improvement procedure and get out of local optimum traps. Then, a procedure is applied starting from the selected solution $S^{\prime}$ in order to obtain a new local optimum $S^{\prime \prime}$. The final step allows to guide the search while exploring the solution space. More specifically, the neighborhood change step changes the neighborhood or continues in generating neighbors. Several neighborhood change procedures exist, but the widely used is sequential neighborhood step: if the local optimum $S^{\prime \prime}$ is better than the current solution $S$, then $S^{\prime \prime}$ becomes the current solution and the process starts again at the first step with the first neighborhood structure otherwise, the search is resumed in the next neighborhood structure (according to a predefined order) of the 
current solution $S$. The VNS algorithm stops whenever the stopping criterion is fulfilled. In this paper, the stopping criterion is reached whenever the current solution cannot be improved after testing all neighborhood structures in the course of a maximum number of iterations $n_{\max }$.

Many variants of VNS are deduced from this basic scheme [16]. In this paper, we focus on the widely used variants of VNS: basic VNS and general VNS.

\section{Basic VNS (BVNS)}

The BVNS parameters are: an initial solution (generated with a FCFS rule in this study) and a sorted list of neighborhood operators. Through each iteration, this algorithm executes the shaking step, the local search procedure (presented in Algorithm 1) and the neighborhood change step. These ones occur alternately until the current solution is not improved anymore in the course of a maximum number of iterations $n_{\max }$. The algorithm of the BVNS is given in Algorithm 2. In this strategy, $S$ denotes the current solution which represents the best solution found at a given time. $S^{\prime}$ indicates a random solution used in the local search. The solution proposed by the local search $S^{\prime \prime}$ is a candidate solution that may become the current solution.

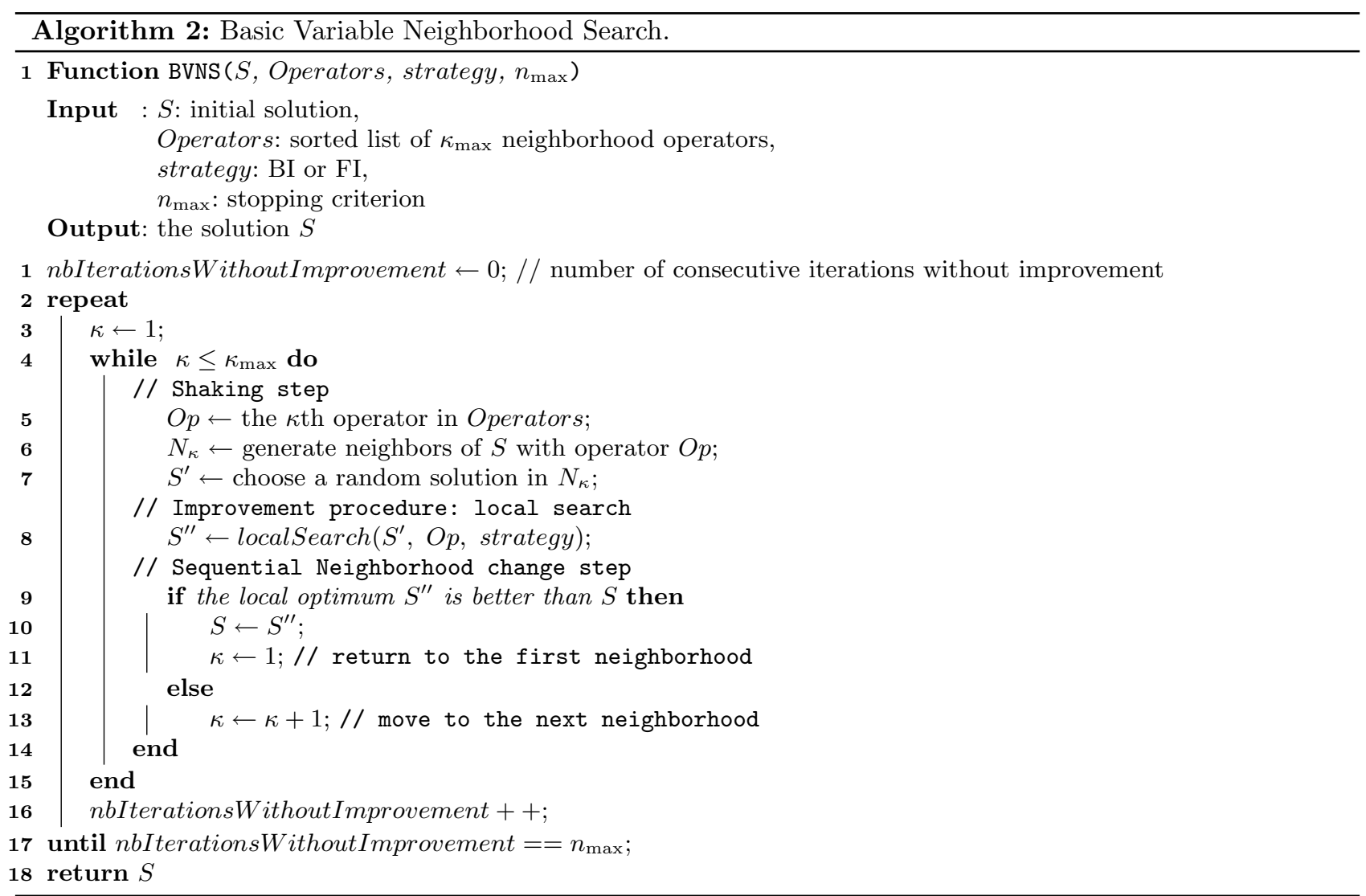

\section{General VNS (GVNS)}

Contrary to the BVNS, the GVNS uses a Variable Neighborhood Descent (VND) procedure instead of a local search. This function requires as parameters an initial solution $S$ (generated with a FCFS rule in this study) and two sorted lists of neighborhood operators. One of them is used within the shaking step and the other one within the VND procedure. To simplify the study hereafter, these two sorted lists will be the same. 
The algorithm of the GVNS is given in Algorithm 3. Several variants of VND exist in the literature and some of them were compared in [24]. In this study, two procedures stood out from the others: the basic sequential VND (B-VND) and the union VND (U-VND). In our paper, we focused on this two variants. The parameters of these two functions are an initial solution $S$ and a sorted list of neighborhood operators. The B-VND algorithm explores iteratively each neighborhood defined by the current operator according to the established order. The search strategy BI or FI is used to select a solution within a neighborhood. Whenever the current solution is improved, the process starts again at the first neighborhood structure. The algorithm is stopped if the current solution cannot be improved. The U-VND process is the same as the B-VND except that the search is continued in a single neighborhood that is obtained as the union of all predefined neighborhoods. This algorithm is used with BI search strategy, else U-VND is equivalent with B-VND. The algorithms of B-VND and U-VND are given in Algorithm 4 and Algorithm 5 respectively.

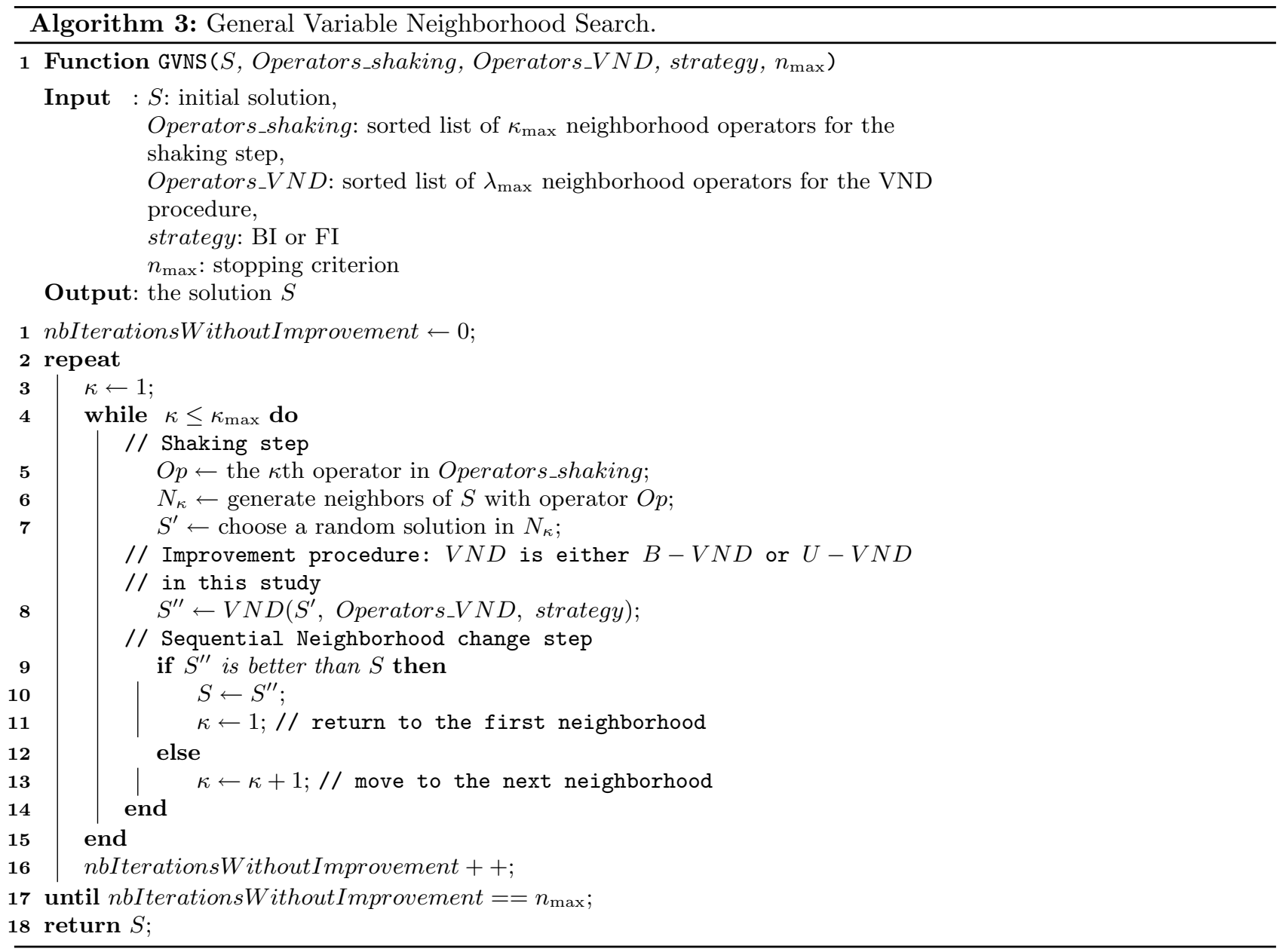

\section{EXPERIMENTAL STUDY}

According to the previous Sections 4 and 5, the VNS algorithms can differ depending on the following configuration aspects (Fig. 2): 

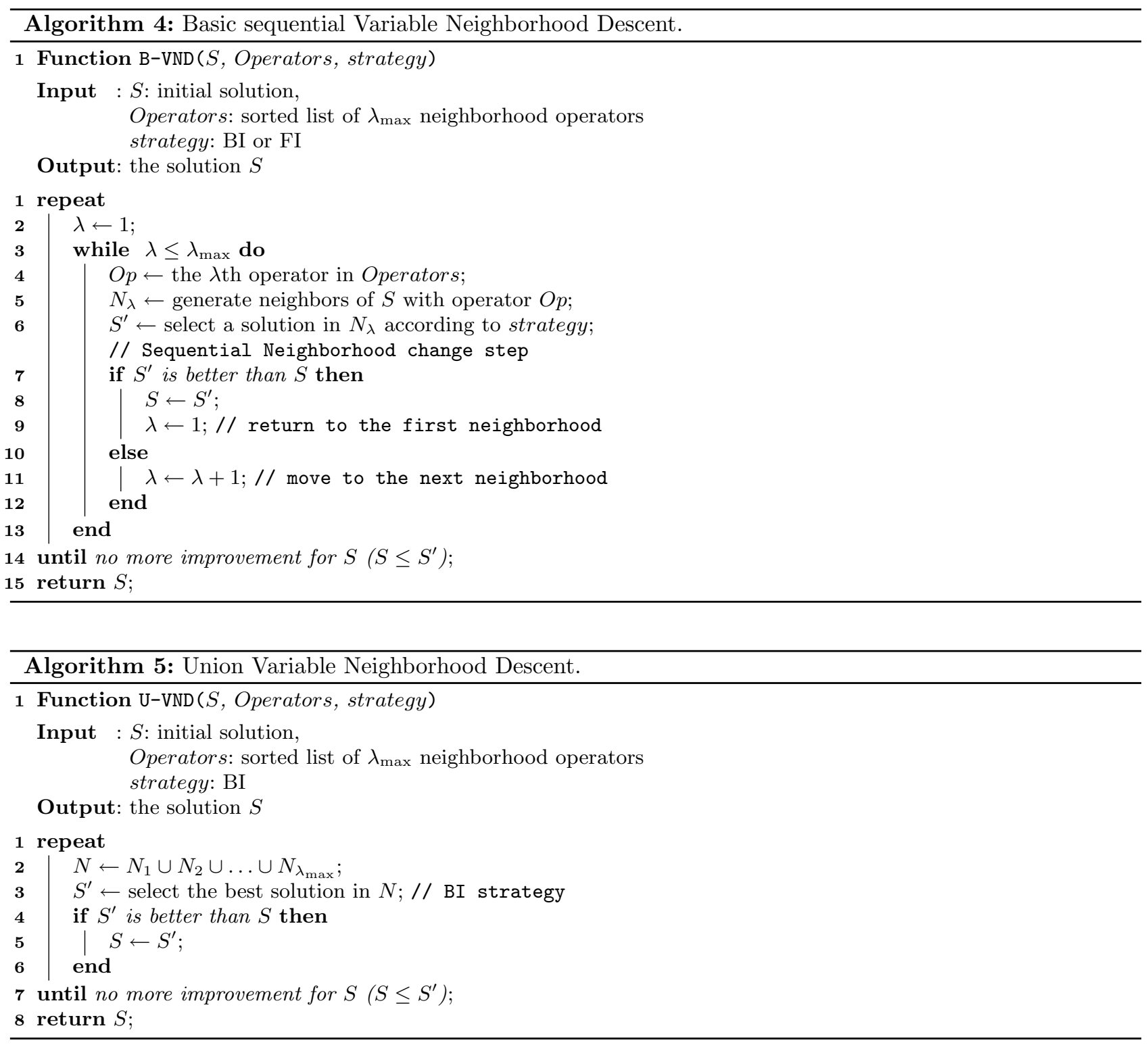

(1) Neighborhood operators: among the proposed operators DEM, TEM, TIM and TIAFDM, which one(s) to use in VNS algorithms? How many? In which order?

(2) Search strategy for the improvement procedure: first (FI) or best improvement (BI)?

(3) Improvement procedure: a simple local search or a VND variant (B-VND or U-VND)? In other words, which VNS variants to be used: BVNS, GVNS using B-VND or GVNS using U-VND?

(4) Stopping criterion: what is the necessary amount of consecutive iterations while the solution remains without improvement? 1,10 or 30 iterations?

We can see that many different VNS variants can be obtained by choosing different settings. We can also think that these choices could have an impact on the quality of the final solution. Hence in the rest of this paper, we conduct several experiments to compare several VNS metaheuristic variants under these different settings. 
The aim of this empirical study is to find the most effective configuration(s) of VNS metaheuristic for solving the cross-dock problem.

This experimental study consists of four parts. In the first part, we evaluate and compare the four neighborhood operators one by one within a local search. These tests are conducted under two different settings: a local search that uses BI search strategy and a local search that uses FI search strategy. The second part is devoted to the way these operators could be combined in a BVNS structure according to their efficiency. We test several strategies that differ by the number and the order of the neighborhood operators. Then, a comparison of VNS variants is performed in the third part. In the last part, we evaluate the influence of the stopping criterion within these variants over the results. In each part of this study, the performance of the tested algorithms is evaluated on various instances of the cross-docking problem. The best performing algorithms are compared with the performance of CPLEX solver. A detailed description of the results is presented in the tables of Appendix A. These results are also visible on the following link https://www . lgi2a.univ-artois.fr/ hsu/Recherche/CROSSDOCK-BENCHS/.

\subsection{Experimental setup and parameter settings}

The computational experiments are conducted on Intel @ Core $\left({ }^{\mathrm{TM}}\right)$ i7 CPU K875 @ $2.93 \mathrm{GHz}$ of a machine using $2.00 \mathrm{~Gb}$ RAM. The runs are made on a 64 bits machine. The instances used are generated in the same way as in [21] and they are sorted by the number of trucks and also the number of docks. In the first row of each table of results in Appendix A, $n \times m$ denotes that there are $n$ trucks and $m$ docks for a group of instances. Each category consists of five instances. In total we have 95 instances (19 groups of instances) ranging from $n=10$ and $m=3$ up to $n=60$ and $m=10$. In our study, each instance has been executed thirty five times and each result cell in the table contains the average value of these runs. As comparison, we use ILOG CPLEX 12.6.3 with default settings to solve the formulation presented in Section 3 with a time limit of $7200 \mathrm{~s}$. The solution quality and computational time of the proposed metaheuristics are evaluated. Hereafter, whenever comparing two methods, the mentioned percentages of improvement of the solution quality (respectively runtime) are determined firstly by evaluating for each instance the ratio between the value of objective function (or runtime) obtained with the first method and the value obtained with the second method. Afterward, we calculate the average of all the objective function ratios (or runtime) for all the instances. To evaluate the statistical significance of our results, the Friedman test, based on [10] has been conducted.

The Friedman test is a non-parametric statistical test used to detect significant differences between the behavior of $k(k \geq 2)$ algorithms through a set of $b$ instances. To perform the Friedman test, the first step is to rank the results $X_{i j}$ of the metaheuristics $j(j \leq k)$ for each instances $i(i \leq b)$ separately. Let $R\left(X_{i j}\right)$ be the rank from 1 to the best performing algorithm and $k$ to the worst. Then, the total summation of squared ranks $A_{2}$ is calculated:

$$
A_{2}=\sum_{i=1}^{b} \sum_{j=1}^{k}\left[R\left(X_{i j}\right)\right]^{2} .
$$

Therefore, for each metaheuristic $j(j \leq k)$ the summation of the rank is computed $R_{j}=\sum_{i=1}^{b} R\left(X_{i j}\right)$, then $B_{2}$ is calculated:

$$
B_{2}=\frac{1}{b} \sum_{j=1}^{k} R_{j}{ }^{2} .
$$

In this test, under the null-hypothesis, which states that all the algorithms behave similarly, the Friedman statistic can be computed as:

$$
T_{2}=\frac{(b-1)\left[B_{2}-b k(k+1)^{2} / 4\right]}{A_{2}-B_{2}}
$$

which is distributed according to an $F$ distribution with $(k-1)$ and $(k-1)(b-1)$ degrees of freedom. If $T_{2}$ is greater than $1-\alpha$ quantile of the $F$ distribution with $(k-1)$ and $(k-1)(b-1)$ degrees of freedom 
( $\alpha$ a significance level), the null-hypothesis is rejected, i.e. the existence significant differences is found. In this case, we proceed with a post-hoc procedure to characterize these differences.

The post hoc test is used to compare algorithms two by two. This test declares the algorithms $i$ and $j$ significantly different if the absolute difference between the summation of the ranks for $i$ and for $j$ is greater than the critical value for the difference with $(b-1)(k-1)$ degrees of freedom and $\alpha$ a significance level.

In this study, we always use a significance level $\alpha$ equals to 0.01 for the Friedman test and the post-hoc tests will be employed for a significant degree $\alpha=0.05$.

\subsection{Results}

\subsubsection{Comparison of neighborhood operator within $L S$}

In this first part, we evaluate one by one the neighborhood operators (DEM, TEM, TIM and TIAFDM presented in Sect. 4) within a local search. For each operator, LS is tested under two different settings: BI and FI search strategies. The purpose is to determine the efficiency of each operator and the influence of the search strategies in term of solution quality and runtime. The results are presented in Tables A.1 and A.2

Let's focus on the search strategies BI and FI. For all operators, considering the average results, we note that BI provides the best and fastest solutions. Because FI picks the first improvement in the neighborhood which can be a solution far away from the best neighbor. Consequently, the local search algorithm is slower in finding the local optima.

Regarding the neighborhood operators with BI strategy, we can deduce:

(1) If we consider the average solutions (see the average results over entire set of instances in column Average of Tab. A.2), TIM provides the best solution quality, DEM is ranked second followed by TEM, and TIAFDM is the worst. This ranking is the same for each group of instances (except for $14 \times 4$ and $14 \times 6$ ).

(2) Regarding the runtime consumed by each operator, if we consider the average solutions, we obtain the following ranking: TIAFDM is the fastest, DEM and TEM are second and third respectively, and TIM is the slowest. This ranking is the same for each group of instances (except for $10 \times 3$ ).

(3) For each group of instances, TIM is the best operator in term of solution quality, but it is slower than the others. In fact, contrary to the other operators, TIM tries to move all trucks (assigned or not) to another dock available or not at the risk of rejecting another truck. Hence, this operator is slower, but it improves the solution quality because the penalty cost can be minimized by trying to assign rejected trucks.

(4) For each group of instances, TIAFDM is the worst operator in term of solution quality, but it is the faster. This behavior is as expected because usually TIAFDM provide a smaller neighborhood than the others and with no many improved solutions. So the search is faster and with lower quality.

To highlight the significant differences between these neighborhood operators, we conduct the Friedman test. Table 1 shows the results of the Friedman test for solution quality and runtime and summarizes the ranking obtained. In this table and the following ones reporting the results of Friedman test, "Average rank" notices the average of ranks obtained by the corresponding metaheuristic according to the solution quality (or the runtime) over the $b$ groups of instances. Similarly, "Summation" notices the sum of the $b$ ranks obtained and of the $b$ squared ranks. We can notice on Table 1 that for both solution quality and runtime, $T_{2}>F_{0.99,3,54}$ i.e. the null hypothesis is rejected. It means that there exists at least one operator for the local search whose performance is different from at least one of other operators. Consequently the post-hoc paired comparisons is performed to know which neighborhood operator outperforms others.

Table 2 shows the results of the post-hoc test for solution quality and runtime. Each cell of this table contains the absolute difference between the summation of the ranks for the operator of the line (indicated in the Tab. 1) and the summation of the ranks for the operator of the column. Symbol "(-)" indicates that the result of the subtraction is negative, that means that the operator of the line provides better performances than the operator of the column. Conversely, the absence of this symbol indicates that the operator of the column outperforms the one of the line. Underlines values indicate the operators are significantly different because these values are greater than the critical value. For example, let's compare the operators DEM and TEM in term of solution 
TABLE 1. Results of the Friedman test for solution quality and runtime of the Local Search (with $k=4, b=19$ and $\alpha=0.01$ ).

\begin{tabular}{|c|c|c|c|c|c|c|c|c|}
\hline & \multicolumn{2}{|c|}{ DEM } & \multicolumn{2}{|c|}{ TEM } & \multicolumn{2}{|c|}{ TIM } & \multicolumn{2}{|c|}{ TIAFDM } \\
\hline & $R\left(X_{b_{1}}\right)$ & $R\left(X_{b_{1}}\right)^{2}$ & $R\left(X_{b_{2}}\right)$ & $R\left(X_{b_{2}}\right)^{2}$ & $R\left(X_{b_{3}}\right)$ & $R\left(X_{b_{3}}\right)^{2}$ & $R\left(X_{b_{4}}\right)$ & $R\left(X_{b_{4}}\right)^{2}$ \\
\hline \multicolumn{9}{|c|}{ Solution quality } \\
\hline Average rank & 2.11 & & 2.89 & & 1 & & 4 & \\
\hline Summation & 40 & 86 & 55 & 161 & 19 & 19 & 76 & 304 \\
\hline \multicolumn{9}{|l|}{ Runtime } \\
\hline Average rank & 2.05 & & 2.95 & & 4 & & 1 & \\
\hline \multirow[t]{2}{*}{ Summation } & 39 & 81 & 56 & 166 & 76 & 304 & 19 & 19 \\
\hline & Solution & quality & & & & & Runtime & \\
\hline$T_{2}$ & 459.79 & & & & & & 884.5 & \\
\hline$F_{0.99,3,54}$ & & & & & & & & \\
\hline
\end{tabular}

TABle 2. Results of the post-hoc test for solution quality and runtime of the Local Search (with $\alpha=0.05$ and 54 degrees of liberty).

\begin{tabular}{lllll}
\hline \hline \multicolumn{5}{c}{ (a) Solution quality } \\
\hline DEM & TEM & TIM & TIAFDM \\
\hline DEM & - & $15(-)$ & $\underline{21}$ & $\underline{36}(-)$ \\
TEM & - & - & $\underline{36}$ & $\underline{\underline{21}}(-)$ \\
TIM & - & - & $\underline{57}(-)$ \\
TIAFDM & - & - & - & - \\
\hline Critical value & \multicolumn{5}{c}{20.99} \\
\hline \multicolumn{7}{r}{} & DEM & (b) Runtime & TEM & TIM & TIAFDM \\
\hline DEM & - & $19(-)$ & $\underline{38}(-)$ & 19 \\
TEM & - & - & $\underline{19}(-)$ & $\underline{38}$ \\
TIM & - & - & - & $\underline{57}$ \\
TIAFDM & - & - & - & - \\
\hline Critical value & & \multicolumn{5}{c}{20.99} \\
\hline
\end{tabular}

quality: $40-55=-15$; hence the absolute value " 15 " following by " $(-)$ " in the cell of the line DEM and column TEM; as $15<20.99$ (the critical value), DEM and TEM have the same performance. Let's compare the quality solution of DEM and TIAFDM now, i.e. the last cell of the line DEM of the table: $40-76=-36 ; 36>20.99$ so DEM and TIAFDM are significantly different; as the subtraction give a negative result, DEM outperforms TIAFDM. In this way, we can notice that TIM outperforms significantly each one of the other operators in term of solution quality, contrary to TIAFDM which is significantly outperformed by all the other operators. In term of runtime, DEM is equivalent to TEM and to TIAFDM. DEM is faster than TIM and TIAFDM outperforms TEM and TIM.

According to these tests and analysis, we can conclude this following ranking of neighborhood operators: TIM is the best operator, followed jointly by DEM and TEM, TIAFDM takes the last place. Even if TIM is the slowest, it needs in average less of $3 \mathrm{~s}$ to propose a solution $26 \%$ better that the one proposed by TIAFDM. 


\subsubsection{Neighborhood operators within BVNS}

This second part is designed to answer to the following questions: how many neighborhood operators is better to use within a basic VNS? In which order? With this in mind, we evaluate BVNS first with two operators and then with three operators. During these tests, we use the three best operators according to the previous part: TIM, DEM and TEM. Also, BVNS algorithm uses BI for the search strategy and stops when the solution cannot be improved, i.e. with $n_{\max }=1$.

Firstly we evaluate BVNS with these six possible orders of the neighborhoods:

- TEM-TIM

- TIM-TEM

- DEM-TIM

- TIM-DEM

- TEM-DEM

- DEM-TEM

The results are presented in Tables A.3 and A.4. Let's focus on the order of the operators. We can notice this following observations:

(1) TEM-TIM versus TIM-TEM: on average solution (see the average results over entire set of instances in column Average of Table A.4) and for each group of instances, TIM-TEM provides better solution quality (except for $60 \times 10$ ). Regarding the runtime on the average results, TEM-TIM is slightly better.

(2) DEM-TIM versus TIM-DEM: DEM-TIM provides provides better solution quality for each group of instances (except for $18 \times 6$ ) but it is slower (except for $14 \times 4$ ). This observation is valid on the average solution.

(3) TEM-DEM versus DEM-TEM: if we consider average results, DEM-TEM is better regarding the solution quality and the runtime. Also, this order is faster for each group of instances (except for $16 \times 6$ and $25 \times 6$ ).

The results of Friedman tests are summarized in Table 3. Obtained tests show that the null hypothesis is rejected for the solution quality and the runtime as well. The results of the post-hoc test is presented in Table 4. Comparing the order of the operators, the performance is the same for solution quality and runtime, except TIM-TEM provides better solution than the reversed order.

According to this analysis, we can conclude that TIM-TEM outperforms the reversed order, just like TIMDEM and DEM-TEM.

When we compare the six ordered lists of operators, if we consider the average results (column Average of Tab. A.4), we can notice:

(1) Regarding the solution quality, we obtain the following ranking: TIM-TEM provides the best solution, followed by TIM-DEM, then TEM-TIM and DEM-TIM, the two last places are assigned to DEM-TEM and finally TEM-DEM.

(2) For each group of instances, the best solution is provided by the ordered lists with TIM as the first operator (except for $18 \times 6$ and $60 \times 10$ ).

(3) Comparing the runtime, the fastest is DEM-TEM followed by the reversed order (TEM-DEM), DEM-TIM and the reversed order are ranked third and fourth respectively, TEM-TIM and TIM-TEM are the slowest ranked in the fifth and the last place respectively.

The statistical tests show that TIM-TEM provides better solutions than the other operators. DEM+TEM (in two-ways) is worse than all the other operators in term of solution quality, but is the fastest.

Actually, based on these observations, the behavior of the operator TIM shows that it is a typical intensification operator. Indeed the intensification seeks to manage the search in the area around the best found combinations by favouring through each step the choice of solutions that belong to best neighborhoods built earlier. Hence, because TIM provides on average the best solution quality combining with the LS, it is able to explore the search space. As a second operator which is the exploration/diversification operator, DEM and TEM perform better, even if TEM seems to be slightly better. Actually, they are able to diversify the solution in 
TABLE 3. Results of the Friedman test for solution quality and runtime of basic VNS with two operators (with $k=6, b=19$ and $\alpha=0.01$ ).

\begin{tabular}{|c|c|c|c|c|c|c|}
\hline & \multicolumn{2}{|c|}{ DEM-TIM } & \multicolumn{2}{|c|}{ TIM-DEM } & \multicolumn{2}{|c|}{ TEM-TIM } \\
\hline & $R\left(X_{b_{1}}\right)$ & $R\left(X_{b_{1}}\right)^{2}$ & $R\left(X_{b_{2}}\right)$ & $R\left(X_{b_{2}}\right)^{2}$ & $R\left(X_{b_{3}}\right)$ & $R\left(X_{b_{3}}\right)^{2}$ \\
\hline \multicolumn{7}{|c|}{ Solution quality } \\
\hline Average rank & 3.21 & & 1.95 & & 3.42 & \\
\hline Summation & 61 & 205 & 37 & 81 & 65 & 239 \\
\hline \multicolumn{7}{|l|}{ Runtime } \\
\hline Average rank & 3.05 & & 4.05 & & 5.53 & \\
\hline \multirow[t]{3}{*}{ Summation } & 58 & 178 & 77 & 317 & 105 & 585 \\
\hline & \multicolumn{2}{|c|}{ TIM-TEM } & \multicolumn{2}{|c|}{ TEM-DEM } & \multicolumn{2}{|c|}{ DEM-TEM } \\
\hline & $R\left(X_{b_{4}}\right)$ & $R\left(X_{b_{4}}\right)^{2}$ & $R\left(X_{b_{5}}\right)$ & $R\left(X_{b_{5}}\right)^{2}$ & $R\left(X_{b_{6}}\right)$ & $R\left(X_{b_{6}}\right)^{2}$ \\
\hline \multicolumn{7}{|c|}{ Solution quality } \\
\hline Average rank & 1.42 & & 5.53 & & 5.47 & \\
\hline Summation & 27 & 45 & 105 & 585 & 104 & 574 \\
\hline \multicolumn{7}{|l|}{ Runtime } \\
\hline Average rank & 5.37 & & 1.89 & & 1.11 & \\
\hline Summation & 102 & 554 & 36 & 70 & 21 & 25 \\
\hline & \multicolumn{4}{|c|}{ Solution quality } & \multicolumn{2}{|c|}{ Runtime } \\
\hline$T_{2}$ & \multicolumn{4}{|c|}{99.72} & \multicolumn{2}{|l|}{272.09} \\
\hline$F_{0.99,5,90}$ & \multicolumn{6}{|c|}{3.23} \\
\hline
\end{tabular}

TABLE 4. Results of the post-hoc test for solution quality and runtime of basic VNS with two operators (with $\alpha=0.05$ and 90 degrees of liberty).

\begin{tabular}{|c|c|c|c|c|c|c|}
\hline \multicolumn{7}{|c|}{ (a) Solution quality } \\
\hline & $\begin{array}{l}\text { DEM- } \\
\text { TIM }\end{array}$ & $\begin{array}{l}\text { TIM- } \\
\text { DEM }\end{array}$ & $\begin{array}{l}\text { TEM- } \\
\text { TIM }\end{array}$ & $\begin{array}{l}\text { TIM- } \\
\text { TEM }\end{array}$ & $\begin{array}{l}\text { TEM- } \\
\text { DEM }\end{array}$ & $\begin{array}{l}\text { DEM- } \\
\text { TEM }\end{array}$ \\
\hline DEM-TIM & - & 24 & $4(-)$ & $\underline{34}$ & $\underline{44}(-)$ & $\underline{43}(-)$ \\
\hline TIM-DEM & - & - & $28(-)$ & $\overline{10}$ & $\underline{68}(-)$ & $\underline{67}(-)$ \\
\hline TEM-TIM & - & - & - & $\underline{38}$ & $\underline{40}(-)$ & $\underline{39}(-)$ \\
\hline TIM-TEM & - & - & - & $\overline{-}$ & $\underline{\overline{78}}(-)$ & $\overline{77}(-)$ \\
\hline TEM-DEM & - & - & - & - & - & $\overline{1}$ \\
\hline DEM-TEM & - & - & - & - & - & - \\
\hline \multicolumn{2}{|c|}{ Critical value } & \multicolumn{5}{|c|}{33.85} \\
\hline \multicolumn{7}{|c|}{ (b) Runtime } \\
\hline & $\begin{array}{l}\text { DEM- } \\
\text { TIM }\end{array}$ & $\begin{array}{l}\text { TIM- } \\
\text { DEM }\end{array}$ & $\begin{array}{l}\text { TEM- } \\
\text { TIM }\end{array}$ & $\begin{array}{l}\text { TIM- } \\
\text { TEM }\end{array}$ & $\begin{array}{l}\text { TEM- } \\
\text { DEM }\end{array}$ & $\begin{array}{l}\text { DEM- } \\
\text { TEM }\end{array}$ \\
\hline DEM-TIM & - & $19(-)$ & $\underline{47}(-)$ & $\underline{44}(-)$ & 22 & $\underline{37}$ \\
\hline TIM-DEM & - & - & $\overline{28}(-)$ & $\overline{25}(-)$ & $\underline{41}$ & $\overline{56}$ \\
\hline TEM-TIM & - & - & - & 3 & $\underline{69}$ & $\underline{84}$ \\
\hline TIM-TEM & - & - & - & - & $\underline{66}$ & $\underline{81}$ \\
\hline TEM-DEM & - & - & - & - & - & $\overline{15}$ \\
\hline DEM-TEM & - & - & - & - & - & - \\
\hline Critical value & & & & 33.85 & & \\
\hline
\end{tabular}


TABLE 5. Results of the Friedman test for solution quality and runtime of the VNS algorithms with three operators (with $k=4, b=19$ and $\alpha=0.01$ ).

\begin{tabular}{|c|c|c|c|c|c|c|c|c|}
\hline & \multicolumn{2}{|c|}{$\begin{array}{c}\text { BVNS_- } \\
\text { TIMTEMDEM }\end{array}$} & \multicolumn{2}{|c|}{$\begin{array}{c}\text { BVNS_ } \\
\text { TIMDEMTEM }\end{array}$} & \multicolumn{2}{|c|}{$\begin{array}{l}\text { GVNS+ } \\
\text { B-VND }\end{array}$} & \multicolumn{2}{|c|}{$\begin{array}{l}\text { GVNS+ } \\
\text { U-VND }\end{array}$} \\
\hline & $R\left(X_{b_{1}}\right)$ & $R\left(X_{b_{1}}\right)^{2}$ & $R\left(X_{b_{2}}\right)$ & $R\left(X_{b_{2}}\right)^{2}$ & $R\left(X_{b_{3}}\right)$ & $R\left(X_{b_{3}}\right)^{2}$ & $R\left(X_{b_{4}}\right)$ & $R\left(X_{b_{4}}\right)^{2}$ \\
\hline \multicolumn{9}{|c|}{ Solution quality } \\
\hline Average rank & 2.58 & & 2.74 & & 1.21 & & 3.47 & \\
\hline Summation & 49 & 141 & 52 & 150 & 23 & 33 & 66 & 246 \\
\hline \multicolumn{9}{|l|}{ Runtime } \\
\hline Average rank & 1.74 & & 1.32 & & 4 & & 2.95 & \\
\hline \multirow[t]{2}{*}{ Summation } & 33 & 63 & 25 & 37 & 76 & 304 & 56 & 166 \\
\hline & \multicolumn{4}{|c|}{ Solution quality } & & & \multicolumn{2}{|c|}{ Runtime } \\
\hline$T_{2}$ & \multicolumn{4}{|c|}{20.68} & & & \multicolumn{2}{|l|}{141.26} \\
\hline$F_{0.99,3,54}$ & & & & & & & & \\
\hline
\end{tabular}

order to escape local optima and to explore the search space because of the large neighborhood size that TEM and DEM provide. Finally, we can also conclude that TIM-TEM is the best combinaison for BVNS. Hereafter, the latter will be called BVNS_TIMTEM.

In the second step of this part, we decide to evaluate the BVNS algorithm with three operators. Because we showed that TIM is an intensification operator previously, TIM is placed as the first operator. Hence, we compare BVNS using the ordered lists TIM-TEM-DEM (hereafter called BVNS_TIMTEMDEM) and TIM-DEM-TEM (called BVNS_TIMDEMTEM). The results are presented in Tables A.5 and A.6. If we consider the average results (see column Average of Tab. A.6), we can notice:

(1) BVNS_TIMTEMDEM provides better solution quality than BVNS_TIMDEMTEM but it is slightly slower. The statistical analysis shows that BVNS_TIMTEMDEM and BVNS_TIMDEMTEM have the same performance in term of both solution quality and runtime (Tabs. 5 and 6).

(2) When we compare with BVNS_TIMTEM, the last one provides the worst solution quality but it is faster than BVNS using three operators. In fact, adding an operator in BVNS increases the runtime of the algorithm, but allows exploring further the search space.

We can conclude that the list TIM-TEM-DEM in this order is the best sequence for BVNS among all those tested. Even if this one is the slowest, it needs in average less of $14 \mathrm{~s}$ to perform.

\subsubsection{Comparaison with GVNS}

In this third experimental part, we combine the operators TIM-TEM-DEM in this order in another variant of the VNS algorithm: the GVNS. First, we use GVNS with the B-VND (hereafter called GVNS+B-VND), then GVNS is combined with the U-VND (hereafter called GVNS+U-VND). In these two scenarios, GVNS and VND algorithms take as parameters the same list of neighborhood operators (TIM-TEM-DEM). Like BVNS in the previous experiments, GVNS algorithm stops when the solution cannot be improved, i.e. with $n_{\max }=1$.

From the results presented in Tables A.5 and A.6, we can notice if we consider the average results (see column Average of Tab. A.6):

(1) GVNS+B-VND provides better solution quality than GVNS+U-VND but it is slower. This observation is valid for each group of instances (except for the solution quality in $16 \times 4$ ). The good quality of GVNS+BVND regarding to GVNS+U-VND is due to the fact that union VND algorithm's policy is to seek a local optimum within a same large neighborhood obtained here as the union of three neighborhoods. Thus, it can be stuck in local optima. 
TABLE 6 . Results of the post-hoc test for solution quality and runtime of the VNS algorithms with three operators (with $\alpha=0.05$ and 54 degrees of liberty).

\begin{tabular}{|c|c|c|c|c|}
\hline \multicolumn{5}{|c|}{ (a) Solution quality } \\
\hline & $\begin{array}{l}\text { BVNS_ } \\
\text { TIMTEMDEM }\end{array}$ & $\begin{array}{l}\text { BVNS }_{-} \\
\text {TIMDEMTEM }\end{array}$ & $\begin{array}{l}\text { GVNS+ } \\
\text { B-VND }\end{array}$ & $\begin{array}{l}\text { GVNS+ } \\
\text { U-VND }\end{array}$ \\
\hline BVNS_TIMTEMDEM & - & $3(-)$ & $\underline{26}$ & $17(-)$ \\
\hline BVNS_TIMDEMTEM & - & - & $\underline{29}$ & $14(-)$ \\
\hline GVNS+B-VND & - & - & $\overline{-}$ & $\underline{43}(-)$ \\
\hline GVNS+U-VND & - & - & - & - \\
\hline Critical value & \multicolumn{4}{|c|}{20.99} \\
\hline & $\begin{array}{l}\text { BVNS_ } \\
\text { TIMTEMDEM }\end{array}$ & $\begin{array}{l}\text { BVNS }_{-} \\
\text {TIMDEMTEM }\end{array}$ & $\begin{array}{l}\text { GVNS+ } \\
\text { B-VND }\end{array}$ & $\begin{array}{l}\text { GVNS+ } \\
\text { U-VND }\end{array}$ \\
\hline BVNS_TIMTEMDEM & - & 8 & $\underline{43}(-)$ & $23(-)$ \\
\hline BVNS_TIMDEMTEM & - & - & $\underline{51}(-)$ & $\underline{31}(-)$ \\
\hline GVNS+B-VND & - & - & - & $\overline{20}$ \\
\hline GVNS+U-VND & - & - & - & - \\
\hline Critical value & \multicolumn{4}{|c|}{20.99} \\
\hline
\end{tabular}

(2) Comparing GVNS variants and BVNS on the solution quality, we obtain the following ranking: GVNS+BVND provides the best solution quality, BVNS_TIMTEMDEM is ranking second, and GVNS+U-VND is the worst. GVNS+U-VND is the worst because at each iteration, a local optimum is generated with the respect to three neighborhoods structure together, whereas BVNS_TIMTEMDEM uses one operator only that can be different at a next iteration. So the last one has higher probability to get out of a local optima. In the same way and because GVNS+B-VND uses B-VND, it enables to find a local optimum that is more likely to be a global optimum.

(3) Regarding the runtime, the ranking is as follows: BVNS_TIMTEMDEM is the fastest, the second is GVNS+U-VND and the slowest is GVNS+B-VND. In the same way, using several neighborhood structures to search a local optimum consumes more time than with a single neighborhood structure.

The statistical analysis validates these observations. Table 5 summarizes Friedman results for solution quality and runtime. In the two cases, the null hypothesis is rejected. The post-hoc results (see Tab. 6) show that GVNS+B-VND is better in term of solution quality than GVNS+U-VND. GVNS+B-VND outperforms each one of the other variants in term of solution quality, but GVNS+B-VND and GVNS+U-VND are the slowest.

In conclusion, GVNS+B-VND using TIM-TEM-DEM as ordered list provides the best solution quality, but it is the slowest (it is 2 times slower than BVNS_ TIMTEMDEM). However, the runtime consumed by this algorithm is still reasonable because it needs in average $28 \mathrm{~s}$ to perform. So, all the previous experiments lead to the ranking for the three best strategies: GVNS+B-VND is at the head, followed by BVNS_TIMTEMDEM, while GVNS+U-VND is ranking as third.

\subsubsection{Influence of the stopping criterion}

The GVNS algorithm described in Section 5 stops whenever the solution cannot be improved through $n_{\max }$ iterations. In our analysis above, we chose to set $n_{\max }$ to 1 . In this last part of the tests, we evaluate the influence of this parameter on the solution quality by increasing the value of $n_{\max }$. To realize this experiment, we use our best metaheuristic GNVS+B-VND until now and we compare the results obtained with $n_{\max }$ set to 1 , then to 10 (called GNVS+B-VND_10) and to 30 (GNVS+B-VND_30).

From the results presented in Tables A.5 and A.6, we can see:

(1) Regarding the solution quality, for each instance we obtain always the followed ranking: GNVS+B-VND_30 is the best, GNVS+B-VND_10 is ranked in second, while GNVS+B-VND is the worst. 
TABLE 7. Results of the Friedman test for solution quality of the VNS algorithms with $n_{\max } \in$ $\{1 ; 10 ; 30\}$ (with $k=3, b=19$ and $\alpha=0.01$ ).

\begin{tabular}{|c|c|c|c|c|c|c|}
\hline & \multicolumn{2}{|c|}{ GVNS+B-VND } & \multicolumn{2}{|c|}{ GVNS+B-VND_10 } & \multicolumn{2}{|c|}{ GVNS+B-VND_30 } \\
\hline & $R\left(X_{b_{1}}\right)$ & $R\left(X_{b_{1}}\right)^{2}$ & $R\left(X_{b_{2}}\right)$ & $R\left(X_{b_{2}}\right)^{2}$ & $R\left(X_{b_{3}}\right)$ & $R\left(X_{b_{3}}\right)^{2}$ \\
\hline Average rank & 3 & & 1.97 & & 1.03 & \\
\hline Summation & 57 & 171 & 37.5 & 74.25 & 19.5 & 19.5 \\
\hline$F_{0.99,2,36}$ & & & 5.27 & & & \\
\hline
\end{tabular}

TABLE 8. Results of the post-hoc test for solution quality of the VNS algorithms with $n_{\max } \in$ $\{1 ; 10 ; 30\}$ (with $\alpha=0.05$ and 36 degrees of liberty).

\begin{tabular}{llll}
\hline \hline & GVNS+B-VND & GVNS+B-VND_10 & GVNS+B-VND_30 \\
\hline GVNS+B-VND & - & $\underline{19.5}$ & $\underline{37.5}$ \\
GVNS+B-VND_10 & - & - & $\underline{18}$ \\
GVNS+B-VND_30 & - & - & - \\
\hline Critical value & & & 14.76 \\
\hline
\end{tabular}

(2) Regarding the runtime, for each instance, the ranking is in the reversed order than the previous one: GNVS+B-VND is the fastest, GNVS+B-VND_10 stays in second place, and GNVS+B-VND_30 is the slowest.

Friedman test is applied to the results of these three variants. The results are summarized in Table 7 . In term of solution quality, the null hypothesis is rejected. The post-hoc analysis (see Tab. 8) shows that GNVS+B-VND is outperformed by the other two, but in particular GNVS+B-VND_30 provides the best solutions. These results can be explained easily. The more the GVNS algorithm continues its search, the more it can escape the local optima thanks to the shaking step and explore the search space. So, the algorithm has more opportunity to find the optimal solution. However, the procedure surely consumes more CPU times. In our study, with $n_{\max }$ set to 30, GVNS takes in average less than 5 min (less than $1 \mathrm{~s}$ for small instances and up to 40 min for the biggest instances). To solve the cross-dock issue as an operational problem, this runtime is still reasonable.

Figure 7 describes the distribution of studied algorithms according to their performance. The values are the total of the objective functions and the total runtime for all instances. Using the Pareto front, non dominated approaches can be deduced.

\subsection{Comparison with exact method}

Now that we compared different possible configurations for VNS variants to solve the cross-dock problem, we evaluate the performances of the best algorithms (i.e. BVNS_TIMTEMDEM, GVNS+B-VND variants and GVNS+U-VND) with those of CPLEX configured with a time limit of $7200 \mathrm{~s}$.

Let's focus on small instances (from $10 \times 3$ to $18 \times 4$ ). We can observe:

(1) CPLEX always finds optimal solution in less than two hours. However, for each group of instances, CPLEX is slower than the VNS variants.

(2) Our best VNS variants can get near optimal solutions in a much shorter time than CPLEX. Table 9, shows the average gap expressed in percentage of the objective function values between the best algorithms and CPLEX for the small instances. Each percentage is calculated by averaging the gaps for the small instances presented in the tables of Appendix A for the corresponding algorithm. This gap is less than 1\%, and goes down $0.3 \%$ with GVNS+B-VND_30. 


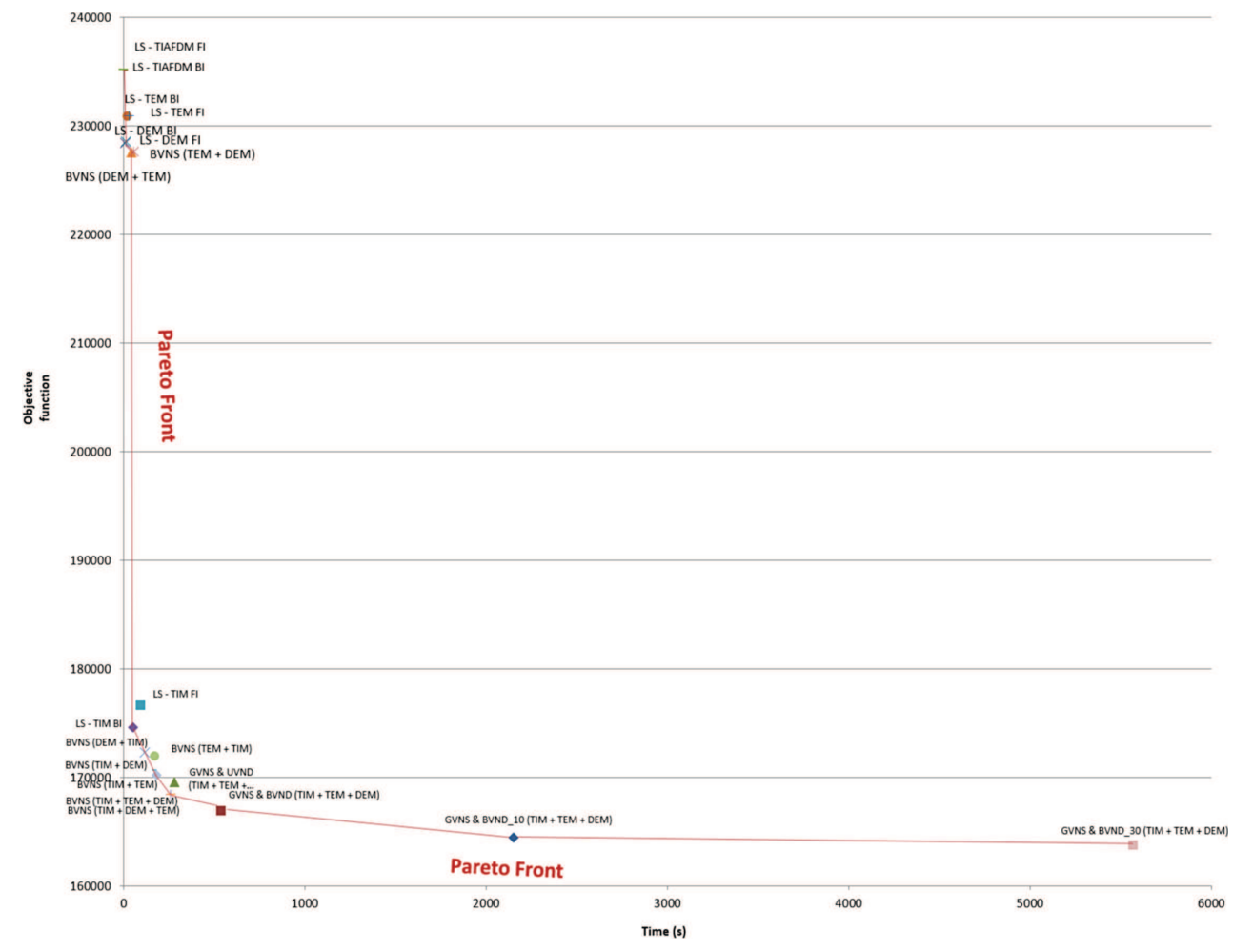

FiguRE 7. Metaheuristics distribution according to their performance with Pareto front drawing.

TABLE 9. Average gap of the objective value with CPLEX on the small instances (from $10 \times 3$ to $18 \times 4$ ) expressed in percentage.

\begin{tabular}{|c|c|c|c|c|c|c|c|c|}
\hline \multirow[b]{2}{*}{ LS } & \multicolumn{2}{|r|}{ TIM } & \multicolumn{2}{|r|}{ DEM } & \multicolumn{2}{|r|}{ TEM } & \multicolumn{2}{|r|}{ TIAFDM } \\
\hline & $\mathrm{BI}$ & FI & $\mathrm{BI}$ & FI & $\mathrm{BI}$ & FI & $\mathrm{BI}$ & FI \\
\hline Gap (\%) & 2.8 & 4.6 & 31 & 31 & 31.2 & 31.3 & 33.3 & 33.3 \\
\hline BVNS & \multicolumn{2}{|c|}{ TIM-DEM } & \multicolumn{2}{|c|}{ DEM-TIM } & \multicolumn{2}{|r|}{ TIM-TEM } & \multicolumn{2}{|r|}{ TEM-TIM } \\
\hline Gap (\%) & \multicolumn{2}{|c|}{1.4} & \multicolumn{2}{|c|}{1.9} & \multicolumn{2}{|c|}{1.4} & \multicolumn{2}{|c|}{2.3} \\
\hline BVNS & \multicolumn{2}{|c|}{ DEM-TEM } & \multicolumn{2}{|c|}{ TEM-DEM } & \multicolumn{2}{|c|}{ BVNS_TIMDEMTEM } & \multicolumn{2}{|c|}{ BVNS_TIMTEMDEM } \\
\hline Gap (\%) & \multicolumn{2}{|c|}{30.7} & \multicolumn{2}{|c|}{30.7} & \multicolumn{2}{|c|}{1} & \multicolumn{2}{|c|}{0.86} \\
\hline GVNS & \multicolumn{2}{|c|}{ GVNS+B-VND } & \multicolumn{2}{|c|}{ GVNS+U-VND } & \multicolumn{2}{|c|}{ GVNS+B-VND_10 } & \multicolumn{2}{|c|}{ GVNS+B-VND_30 } \\
\hline Gap (\%) & \multicolumn{2}{|c|}{0.9} & \multicolumn{2}{|c|}{1.35} & \multicolumn{2}{|c|}{0.4} & \multicolumn{2}{|c|}{0.3} \\
\hline
\end{tabular}

(3) The best VNS variants enable to find at least one time (over the 35 runs) the optimal solution for almost all the instances (see lignes "min" in the table of results of Appendix A).

(4) For each group of instances, GVNS+B-VND_10 and GVNS+B-VND_30 always find at least one time the optimal solution. For some group, they enable to find the optimal solution (hence their gap of 0.4 and 0.3 respectively).

In order to reveal significant differences between CPLEX and the more efficient variants of this study, we apply Friedman test (see Tabs. 10 and 12). We apply this test only to the small instances to allow comparison with 
TABLE 10. Results of the Friedman test for solution quality and runtime of CPLEX and VNS algorithms with three operators (with $k=4, b=8$ and $\alpha=0.01$ ).

\begin{tabular}{|c|c|c|c|c|c|c|c|c|}
\hline & \multicolumn{2}{|c|}{ CPLEX } & \multicolumn{2}{|c|}{$\begin{array}{c}\text { BVNS_ } \\
\text { TIMTEMDEM }\end{array}$} & \multicolumn{2}{|c|}{$\begin{array}{l}\text { GVNS+ } \\
\text { B-VND }\end{array}$} & \multicolumn{2}{|c|}{$\begin{array}{l}\text { GVNS+ } \\
\text { U-VND }\end{array}$} \\
\hline & $R\left(X_{b_{1}}\right)$ & $R\left(X_{b_{1}}\right)^{2}$ & $R\left(X_{b_{2}}\right)$ & $R\left(X_{b_{2}}\right)^{2}$ & $R\left(X_{b_{3}}\right)$ & $R\left(X_{b_{3}}\right)^{2}$ & $R\left(X_{b_{4}}\right)$ & $R\left(X_{b_{4}}\right)^{2}$ \\
\hline \multicolumn{9}{|c|}{ Solution quality } \\
\hline Average rank & 1 & & 3.13 & & 2.38 & & 3.5 & \\
\hline Summation & 8 & 8 & 25 & 83 & 19 & 47 & 28 & 102 \\
\hline \multicolumn{9}{|l|}{ Runtime } \\
\hline Average rank & 4 & & 1 & & 3 & & 2 & \\
\hline Summation & 32 & 128 & 8 & 8 & 24 & 72 & 16 & 32 \\
\hline$T_{2}$ & & & \multicolumn{4}{|c|}{$\begin{array}{l}\text { Solution quality } \\
19.05\end{array}$} & \multicolumn{2}{|c|}{ Runtime } \\
\hline$F_{0.99,3,21}$ & & & & & & & & \\
\hline
\end{tabular}

TABLE 11. Results of the post-hoc test for solution quality and runtime of CPLEX and VNS algorithms with three operators (with $\alpha=0.05$ and 21 degrees of liberty).

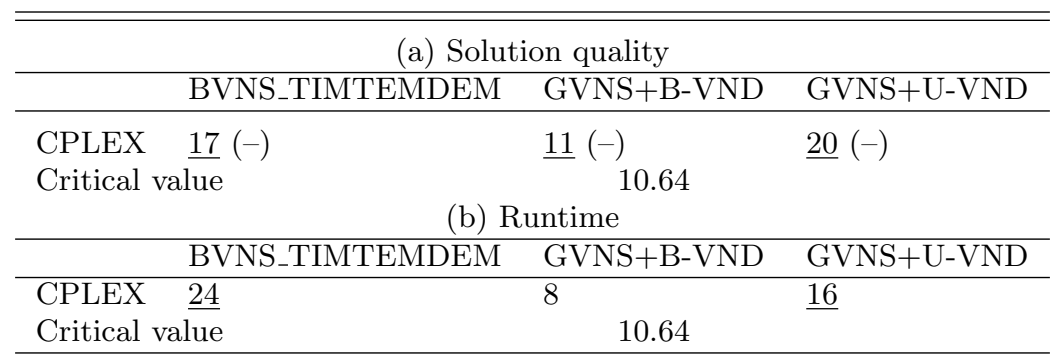

TABLE 12. Results of the Friedman test for solution quality of CPLEX and VNS algorithms with $n_{\max } \in\{1 ; 10 ; 30\}$ (with $k=4, b=8$ and $\alpha=0.01$ ).

\begin{tabular}{|c|c|c|c|c|c|c|c|c|}
\hline & \multicolumn{2}{|c|}{ CPLEX } & \multicolumn{2}{|c|}{$\begin{array}{l}\text { GVNS+ } \\
\text { B-VND }\end{array}$} & \multicolumn{2}{|c|}{$\begin{array}{c}\text { GVNS+ } \\
\text { B-VND_10 }\end{array}$} & \multicolumn{2}{|c|}{$\begin{array}{c}\text { GVNS+ } \\
\text { B-VND_30 }\end{array}$} \\
\hline & $R\left(X_{b_{1}}\right)$ & $R\left(X_{b_{1}}\right)^{2}$ & $R\left(X_{b_{2}}\right)$ & $R\left(X_{b_{2}}\right)^{2}$ & $R\left(X_{b_{3}}\right)$ & $R\left(X_{b_{3}}\right)^{2}$ & $R\left(X_{b_{4}}\right)$ & $\overline{R\left(X_{b_{4}}\right)^{2}}$ \\
\hline Average Rank & 1.25 & & 4 & & 2.88 & & 1.88 & \\
\hline Summation & 10 & 13.5 & 32 & 128 & 23 & 67 & 15 & 28.5 \\
\hline
\end{tabular}

the optimal solutions provided by CPLEX. Both in term of solution quality and runtime, the null hypothesis is rejected. The post-hoc analysis (see Tabs. 11 and 13) shows that CPLEX is the slowest, but in term of solution quality, it outperforms the metaheuristics excluding GVNS+B-VND_30. Indeed, CPLEX and GVNS+B-VND_30 have the same performance.

For the instances $18 \times 6$ and $20 \times 6$, CPLEX does not find the optimal solution in all instances, it provides only lower bounds. For the instance $18 \times 6$, CPLEX outperforms our algorithms, but these last ones are able to find at least one solution with a better quality. For the instances $20 \times 6$, the three GVNS+B-VND variants outperform CPLEX and these algorithms enable to find at least one time an even better solution. 
TABLE 13. Results of the post-hoc test for solution quality of CPLEX and VNS algorithms with $n_{\max } \in\{1 ; 10 ; 30\}$ (with $\alpha=0.05$ and 21 degrees of liberty).

\begin{tabular}{|c|c|c|c|}
\hline & GVNS+B-VND & GVNS+B-VND_10 & GVNS+B-VND_30 \\
\hline $\begin{array}{l}\text { CPLEX } \\
\text { Critical value }\end{array}$ & $\underline{22}(-)$ & $\underline{13}(-)$ & $5(-)$ \\
\hline
\end{tabular}

For large instances (from $20 \times 8$ ), CPLEX is not able to provide optimal solution in less than two hours. Whereas, our best algorithms provide near optimal solutions within a computational time between $0.02 \mathrm{~s}$ and 40 min.

This final analysis highlights the efficiency of the best VNS: BVNS_TIMTEMDEM and GVNS+B-VND variants. In particular, GVNS+B-VND_30 is the only one to have the same performance as CPLEX in term of solution quality. We may conclude that to solve the cross-dock problem at best with VNS, it is preferable to perform GVNS combined with a B-VND using Best Improvement procedure and with TIM-TEM-DEM as ordered list of neighborhood operators. If this algorithm stops whenever the solution cannot be improved through at least 30 iterations, GVNS achieves high performance.

\section{Conclusion And PeRspective}

To operate cross-dock successfully, an efficient assignment of trucks to docks is one of the key decisions. In this paper, we sought to find an optimal assignment for each truck within its time window to increase the efficiency of the cross-dock and to minimize the total cost of transferring shipments within the cross-dock and the penalty cost of non-delivering goods.

First, we proposed an amended ILP model based on one proposed by Miao et al. [21]. To get optimal solutions the CPLEX software were used. Since the problem is NP-hard, it was unlikely to optimally solve the problem in a reasonable time.

Hence, we proposed a VNS metaheuristic to solve this problem approximately. Because different VNS variants can be obtained depending on different configurations and setting parameters, the main purpose of this paper is to deliver a wide strucutred experimental study and a sensitivity analysis to compare these variants. The aim was to highlight the most effective VNS configuration for solving the cross-dock problem at best. First of all, we provided four neighborhood operators (TIM, TEM, DEM, TIAFDM) and compared them within a local search so as to illustrate their characteristics and their efficiency. This step allowed also to compare two search strategies: BI versus FI. Then, we tested the influence on the solution quality and runtime of the order of operators within BVNS using two then three operators. Moreover, we compared three VNS variants: BVNS that uses a local search versus GVNS that uses a B-VND versus GVNS that uses U-VND. Finally, we evaluated the influence of the stopping criterion within VNS.

This empirical study provided many answers. First, the behavior of the operator TIM illustrated that it is a typical intensification operator while DEM and TEM perform as a diversification operator; TIM-TEM-DEM in this order combined with the best improvement strategy is most successful for VNS; GVNS using B-VND significantly outperforms BVNS and GVNS using U-VND; increasing the value of $n_{\text {max }}$ for the stopping criterion improves solution quality; GVNS that uses B-VND with $n_{\max }=30$ has the same performance as CPLEX while consuming much less time.

A perspective to this work would be to include other VNS settings in the empirical study, like the initial solution or other VNS variants. An hybridisation schema could be used to solve the cross-dock problem, for example matheuristics based on integrating metaheuristics and exact methods. In addition, we can evolve this problem, for example by taking into consideration the capacity of trucks or considering soft time windows for trucks which have been discarded in the current version of this model. 


\section{Appendix A. TABle of Results}

LS - operator with strategy BI or FI.

VNS variant (first operator $+\ldots+$ last operator) with strategy BI.

For CPLEX results, $O b j$ is the value of the objective function provided in TIME second, and $L B$ is the lower bound given.

For metaheuristics results, $O b j$ is the average value of the objective function, Time is the average runtime in second, $\sigma$ is the standard deviation and $\min$ the value of the smallest objective function. Gap is the difference between the objective function given by the metaheuristic $O b j$ and the lower bound $L B$ given by CPLEX, expressed as a percentage.

The columns Total and Average show respectively the sum and the average results over entire set of instances.

In each table and for each instances group, the best average objective function provided by a metaheuristic is in bold characters, as well as the best average runtime consumed. 


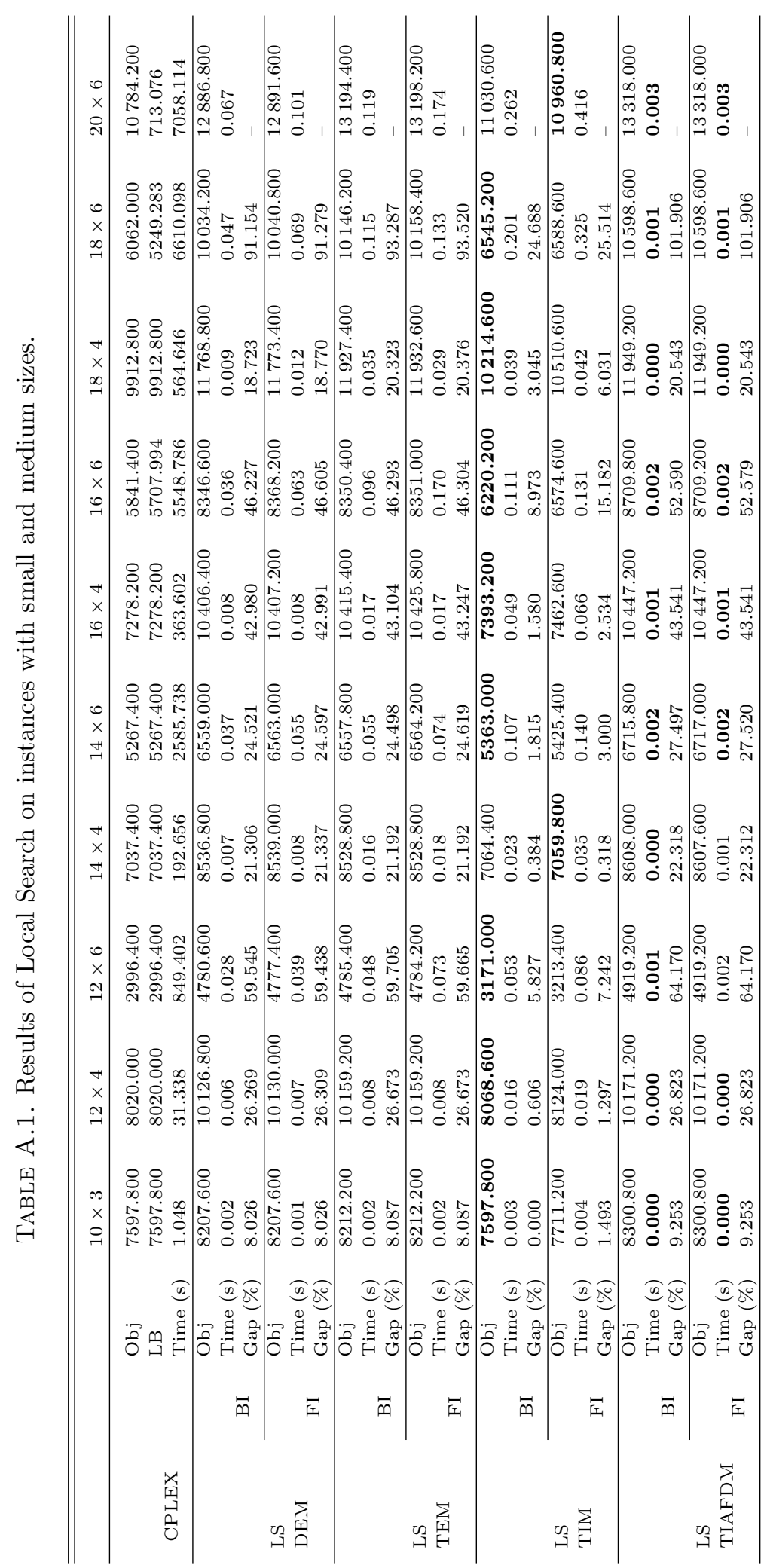




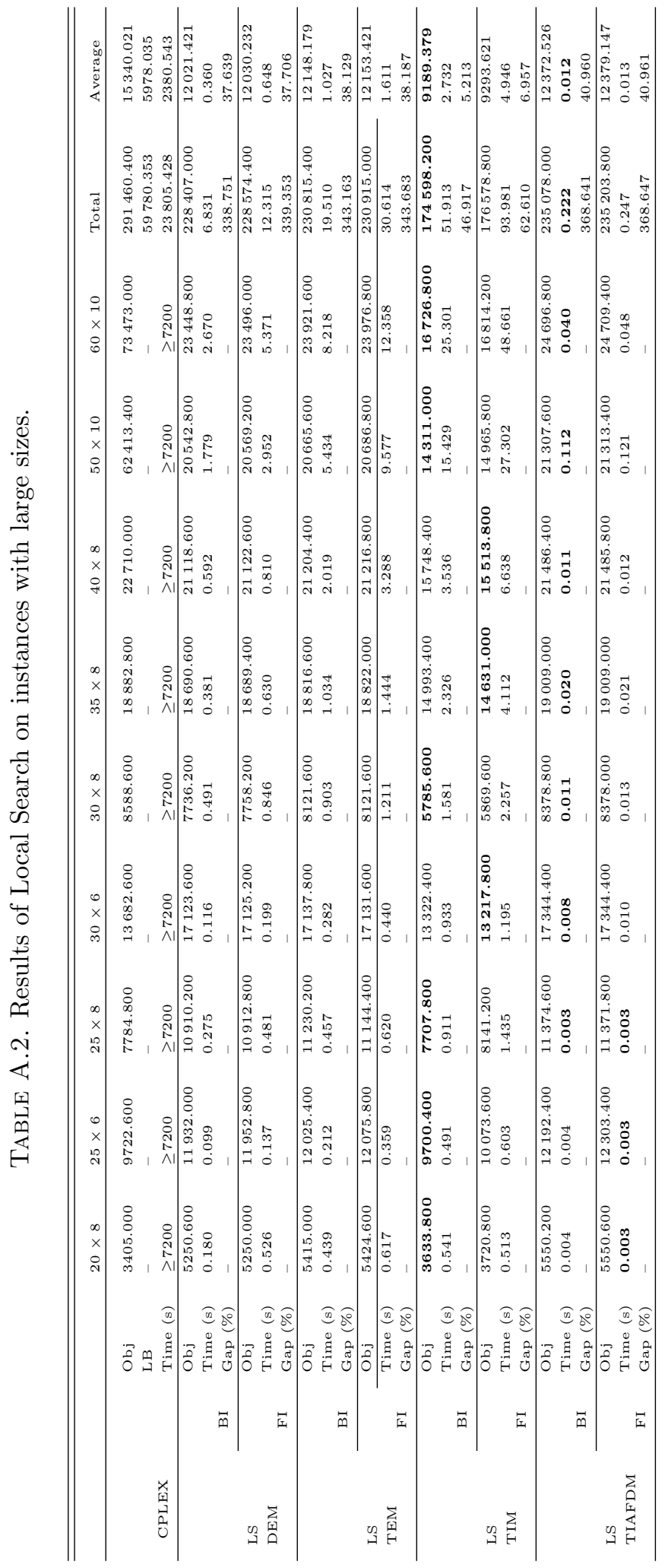




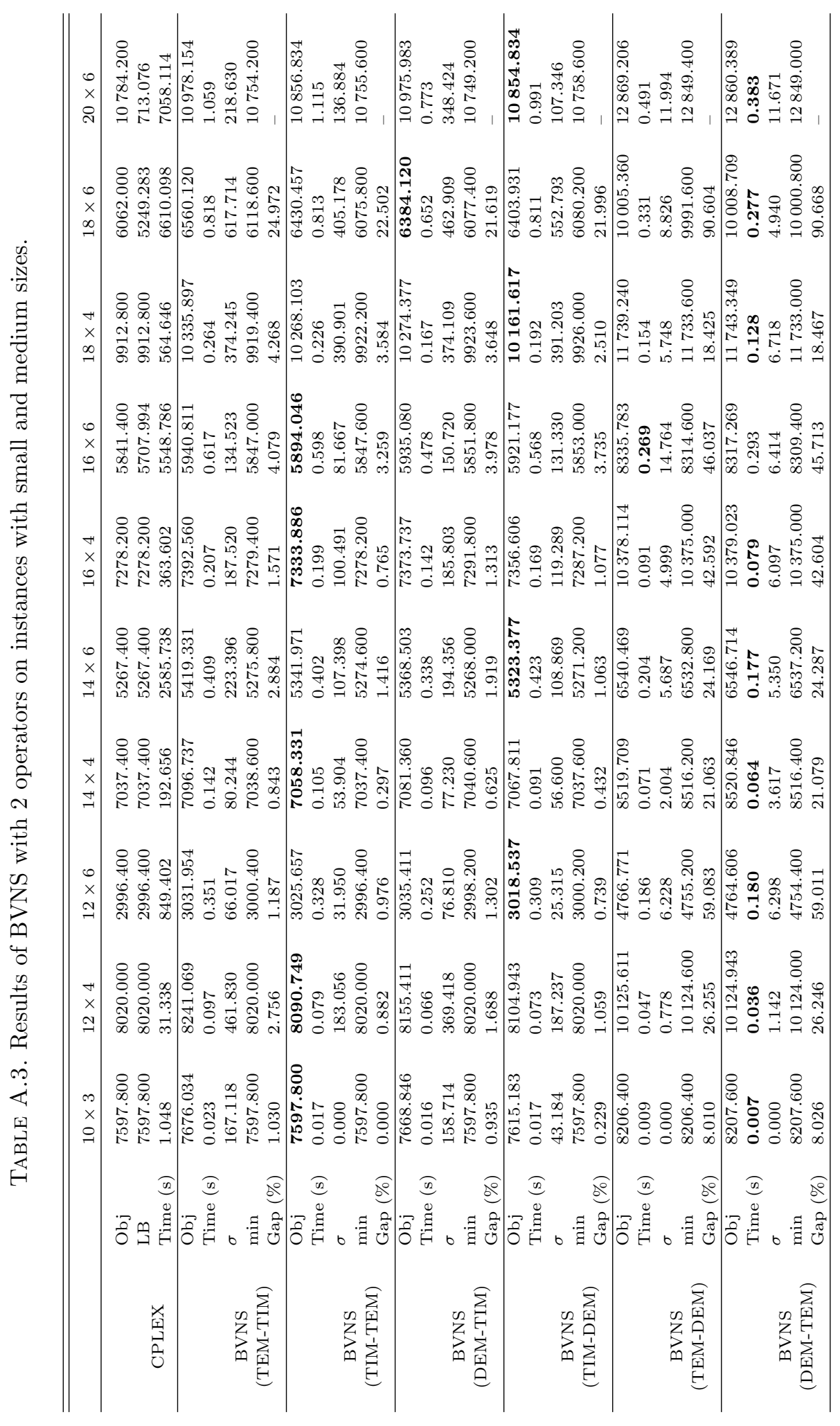




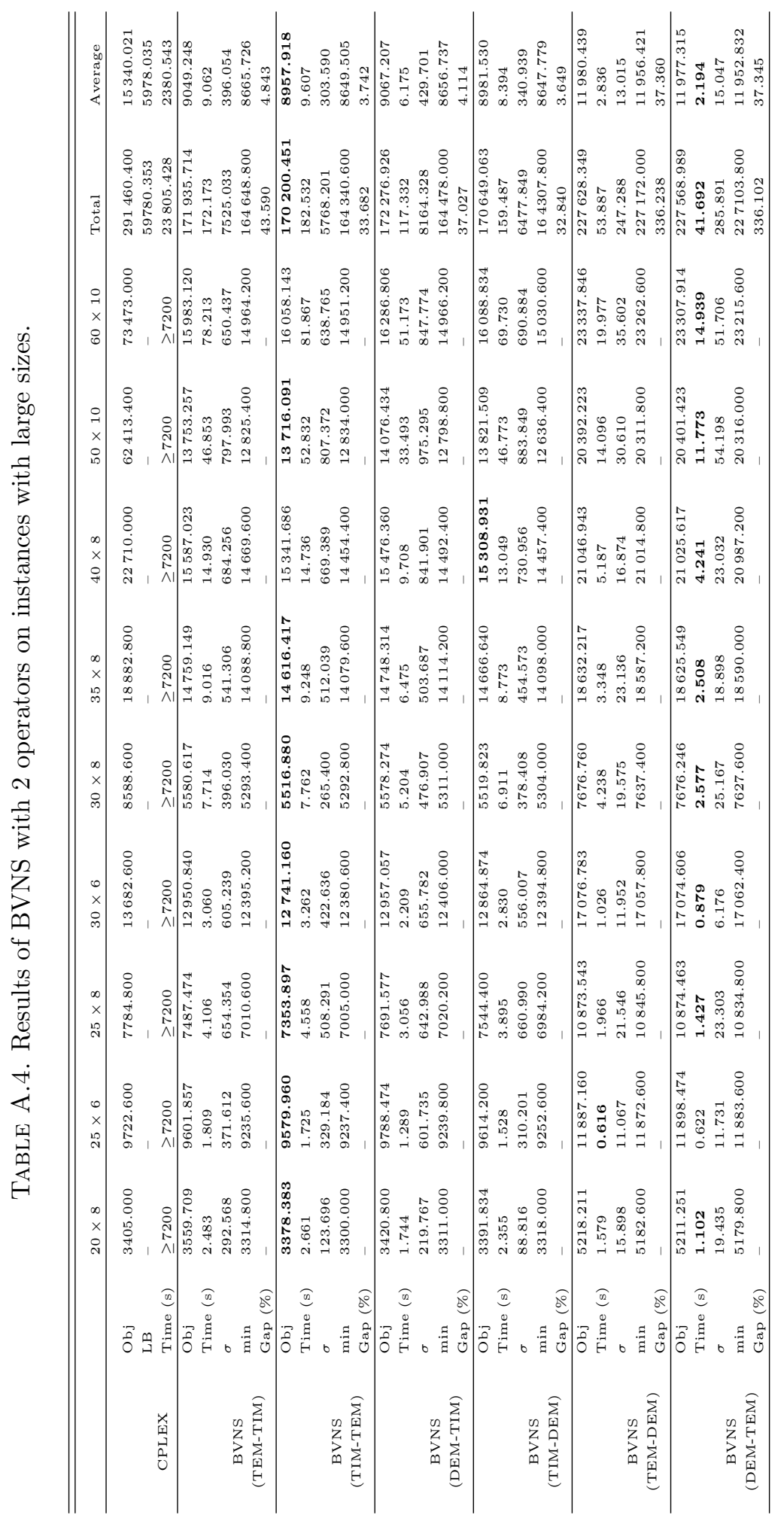




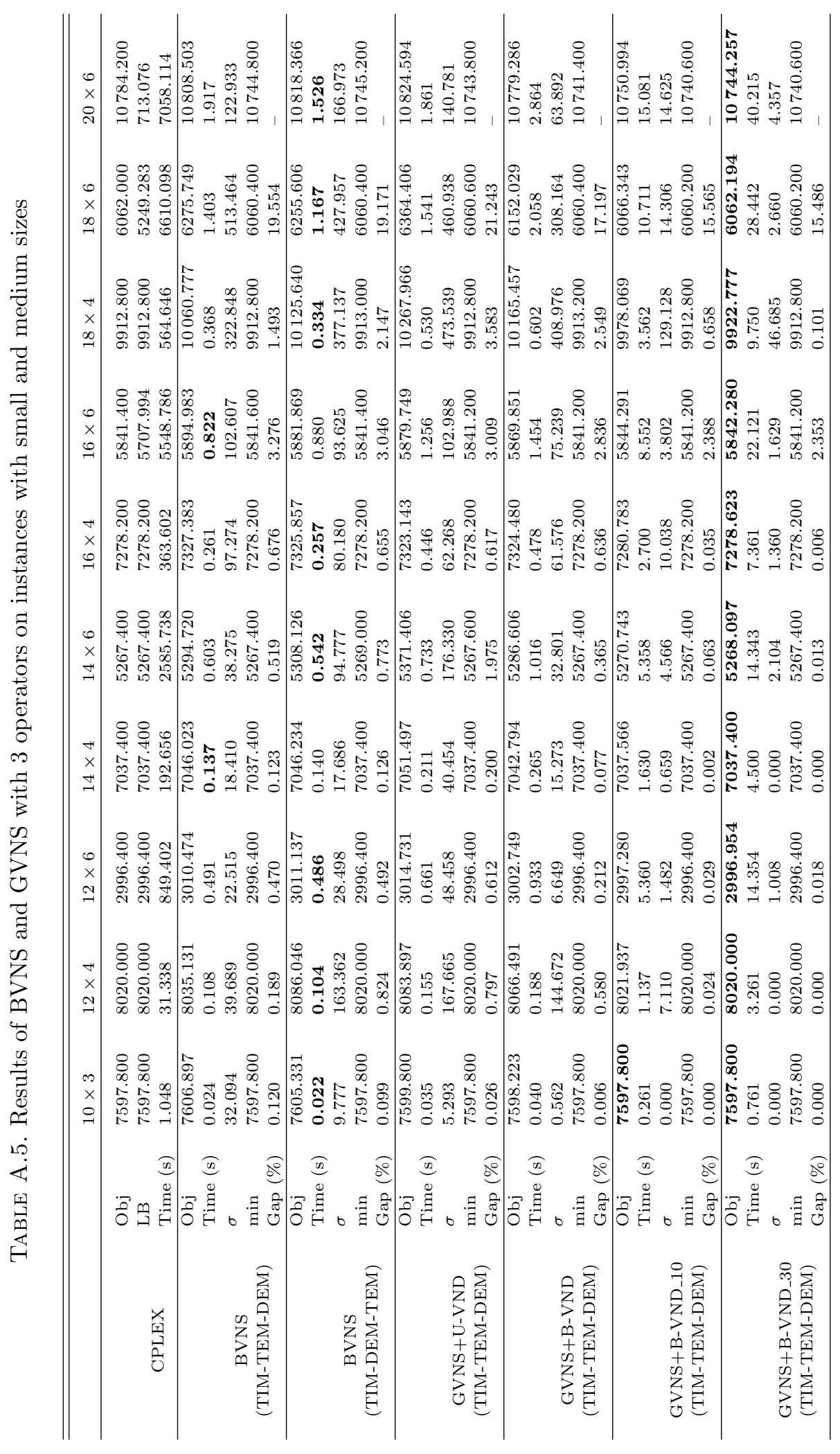




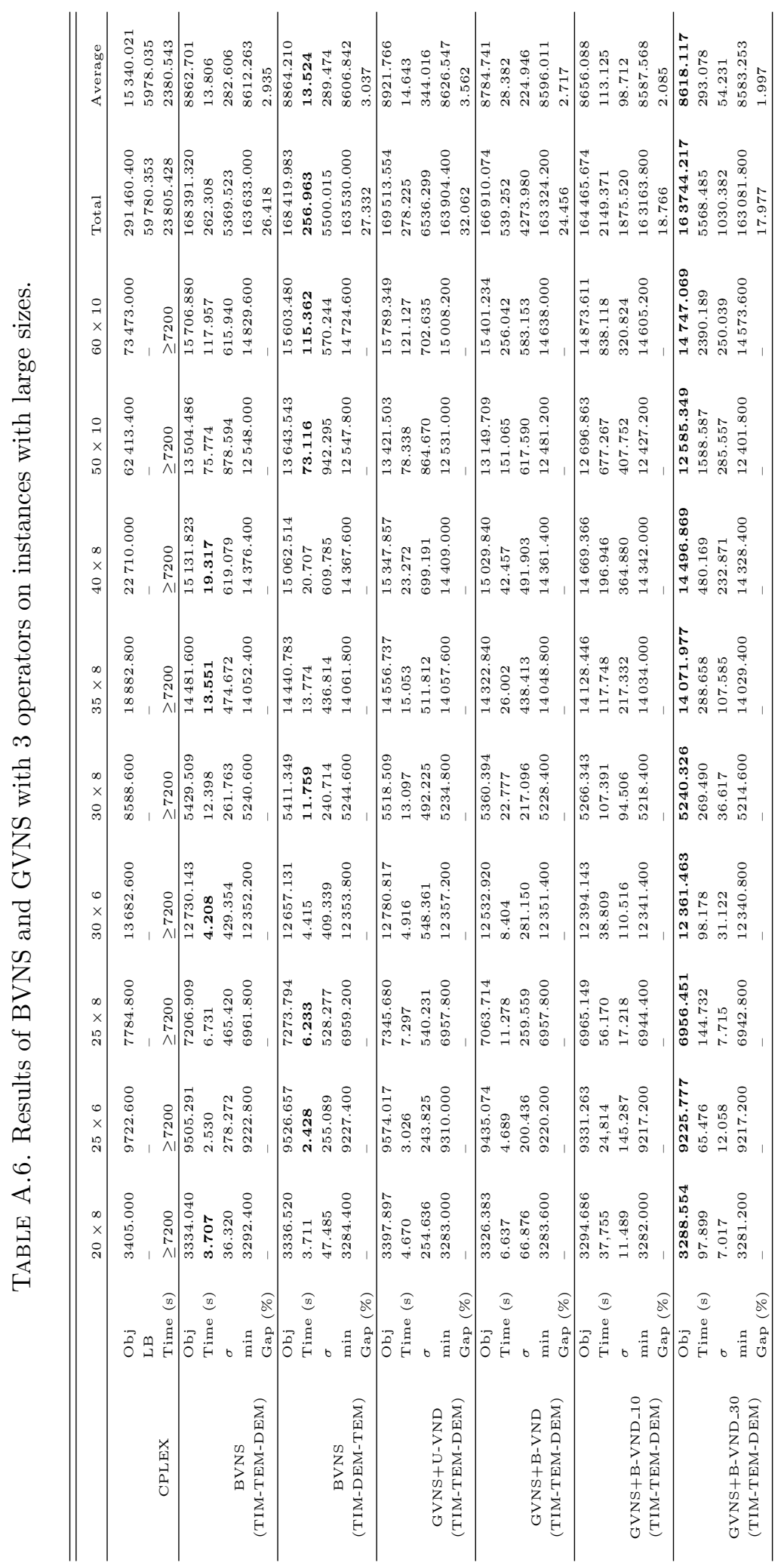


Acknowledgements. This work is a part of the ELSAT2020 project. The ELSAT2020 project is co-financed by the European Union with the European Regional Development Fund, the French state and the Hauts de France Region Council.

\section{REFERENCES}

[1] G. Alpan, A.-L. Ladier, R. Larbi and B. Penz, Heuristic solutions for transshipment problems in a multiple door cross docking warehouse. Comput. Ind. Eng. 61 (2011) 402-408.

[2] G. Alpan, R. Larbi and B. Penz, A bounded dynamic programming approach to schedule operations in a cross docking platform. Comput. Ind. Eng. 60 (2011) 385-396.

[3] U.M. Apte and S. Viswanathan, Effective cross docking for improving distribution efficiencies. Int. J. Logistics 3 (2000) 291-302.

[4] O. Babic, D. Teodorovic and V. Tošic, Aircraft stand assignment to minimize walking. J. Trans. Eng. 110 (1984) 55-66.

[5] A.R. Boloori Arabani, S.M.1T. Fatemi Ghomi and M. Zandieh, Meta-heuristics implementation for scheduling of trucks in a cross-docking system with temporary storage. Expert Syst. App. 38 (2011) 1964-1979.

[6] N. Boysen and M. Fliedner, Cross dock scheduling: Classification, literature review and research agenda. Omega 38 (2010) 413-422.

[7] P. Buijs, I.F.A. Vis and H.J. Carlo, Synchronization in cross-docking networks: a research classification and framework. Eur. J. Oper. Res. 239 (2014) 593-608.

[8] F. Chen and C.-Y. Lee, Minimizing the makespan in a two-machine cross-docking flow shop problem. Eur. J. Oper. Res. 193 (2009) 59-72. ISSN 0377-2217.

[9] Y. Cohen and B. Keren, Trailer to door assignment in a synchronous cross-dock operation. Int. J. Logistics Syst. Manag. 5 (2009) 574-590.

[10] W.J. Conover, The Friedman test. In: Practical Nonparametric Statistics. John Wiley \& Sons, New York, NY (1999) $369-373$.

[11] H. Ding, A. Lim, B. Rodrigues and Y. Zhu, New heuristics for over-constrained flight to gate assignments. J. Oper. Res. Soc. 55 (2004) $760-768$.

[12] H. Ding, A. Lim, B. Rodrigues and Y. Zhu, The over-constrained airport gate assignment problem. Comput. Oper. Res. 32 (2005) $1867-1880$.

[13] R. Dondo and J. Cerdá, The heterogeneous vehicle routing and truck scheduling problem in a multi-door cross-dock system. Comput. Chem. Eng. 76 (2015) 42-62.

[14] G.B. Fonseca, T.H. Nogueira and M.G. Ravetti, A hybrid Lagrangian metaheuristic for the cross-docking flow shop scheduling problem. Eur. J. Oper. Res. 275 (2019) 139-154.

[15] S. Gelareh, R. N. Monemi, F. Semet and G. Goncalves, A branch-and-cut algorithm for the truck dock assignment problem with operational time constraints. Eur. J. Oper. Res. 249 (2016) 1144-1152.

[16] P. Hansen, N. Mladenović, R. Todosijević and S. Hanafi, Variable neighborhood search: basics and variants. EURO J. Comput. Optim. 5 (2017) 423-454.

[17] A.-L. Ladier and G. Alpan, Cross-docking operations: current research versus industry practice. Omega 62 (2016) 145-162.

[18] A. Lim, B. Rodrigues and Y. Zhu, Airport Gate Scheduling with Time Windows. Artif. Intell. Rev. 24 (2005) 5-31.

[19] A. Lim, H. Ma, and Z. Miao, Truck dock assignment problem with operational time constraint within crossdocks, edited by M. Ali and R. Dapoigny. In: Advances in Applied Artificial Intelligence, Vol. 4031 of Lecture Notes in Computer Science. Springer, Berlin-Heidelberg (2006) 262-271.

[20] A. Lim, H. Ma and Z. Miao, Truck dock assignment problem with time windows and capacity constraint in transshipment network through crossdocks. In: Computational Science and Its Applications - ICCSA 2006, Vol. 3982 of Lecture Notes in Computer Science. Springer, Berlin-Heidelberg (2006) 688-697.

[21] Z. Miao, A. Lim and H. Ma, Truck dock assignment problem with operational time constraint within crossdocks. Eur. J. Oper. Res. 192 (2009) 105-115.

[22] Z. Miao, S. Cai and D. Xu, Applying an adaptive tabu search algorithm to optimize truck-dock assignment in the crossdock management system. Expert Syst. App. 41 (2014) 16-22.

[23] Z. Miao, J. Zhang, Y. Lan and R. Su, A two-stage genetic algorithm for the truck-door assignment problem with limited capacity vehicles and storage area. J. Syst. Sci. Syst. Eng. 28 (2019) 285-298.

[24] A. Mjirda, R. Todosijević, S. Hanafi, P. Hansen and N. Mladenović, Sequential variable neighborhood descent variants: an empirical study on the traveling salesman problem. Int. Trans. Oper. Res. 24 (2017) 615-633.

[25] N. Mladenović and P. Hansen, Variable neighborhood search. Comput. Oper. Res. 24 (1997) 1097-1100.

[26] A. Mohtashami, Scheduling trucks in cross docking systems with temporary storage and repetitive pattern for shipping trucks. Appl. Soft Comput. 36 (2015) 468-486.

[27] A. Mohtashami, M. Tavana, F.J. Santos-Arteaga and A. Fallahian-Najafabadi, A novel multi-objective meta-heuristic model for solving cross-docking scheduling problems. Appl. Soft Comput. 31 (2015) 30-47.

[28] W. Nassief, I. Contreras and R. As'ad, A mixed-integer programming formulation and Lagrangean relaxation for the cross-dock door assignment problem. Int. J. Prod. Res. 54 (2016) 494-508.

[29] W. Nassief, I. Contreras and B. Jaumard, A comparison of formulations and relaxations for cross-dock door assignment problems. Comput. Oper. Res. 94 (2018) 76-88. 
[30] T. Obata, The quadratic assignment problem: evaluation of exact and heuristic algorithms. (1979).

[31] S. Sandal, Staging approaches to reduce overall cost in a crossdock environment. Ph.D. thesis, University of Missouri-Columbia, CO (2005).

[32] G.D. Taylor and J.S. Noble, Determination of staging needs in a crossdock environment. In: Proceedings of 2004 Industrial Engineering Research Conference (2004).

[33] L.Y. Tsui and C.-H. Chang, An optimal solution to a dock door assignment problem. Comput. Ind. Eng. 23 (1992) $283-286$.

[34] B. Vahdani and M. Zandieh, Scheduling trucks in cross-docking systems: robust meta-heuristics. Comput. Ind. Eng. 58 (2010) $12-24$.

[35] J. Van Belle, P. Valckenaers and D. Cattrysse, Cross-docking: State of the art. Omega 40 (2012) 827-846.

[36] I.F.A. Vis and K.J. Roodbergen, Positioning of goods in a cross-docking environment. Comput. Ind. Eng. 54 (2008) 677-689.

[37] W. Yu and P.J. Egbelu, Scheduling of inbound and outbound trucks in cross docking systems with temporary storage. Eur. J. Oper. Res. 184 (2008) 377-396. 